\title{
Exact transient full-field analysis of a finite crack subjected to dynamic anti-plane concentrated loadings in anisotropic materials
}

\author{
BY Yi-Shyong Ing ${ }^{1}$ And Chien-Ching $\mathrm{MA}^{2}$ \\ ${ }^{1}$ Department of Aerospace Engineering, Tamkang University, \\ Tamsui, Taipei, Taiwan 251, Republic of China \\ ${ }^{2}$ Department of Mechanical Engineering, National Taiwan University, \\ Taipei, Taiwan 106, Republic of China (ccma@ntu.edu.tw)
}

In this study, the elastodynamic full-field response of a finite crack in an anisotropic material subjected to a dynamic anti-plane concentrated loading with Heavisidefunction time dependence is investigated. A linear coordinate transformation is introduced to simplify the problem. The linear coordinate transformation reduces the anisotropic finite-crack problem to an equivalent isotropic problem. An alternative methodology, different from the conventional superposition method, is developed to construct the reflected and diffracted wave fields. The transient solutions are determined by superposition of two proposed fundamental solutions in the Laplace transform domain. The fundamental solutions to be used are the problems for applying exponentially distributed traction and displacement on the crack faces and along the crack-tip line in the Laplace transform domain, respectively. Exact analytical transient solutions for dynamic shear stresses, displacement and stress-intensity factor are obtained by using the Cagniard-de Hoop method of Laplace inversion and are expressed in explicitly compact formulations. The solutions have accounted for the contributions of all diffracted waves generated from two crack tips. Numerical results for the time history of shear stresses and stress-intensity factors during the transient process are calculated based on analytical solutions and are discussed in detail. The transient solutions of stresses have been shown to approach the corresponding static values after the first eight waves have passed the field point. The dynamic stressintensity factor will reach a maximum value when the incident wave arrives at the crack tip, and remain constant before the first diffracted wave generated from the other crack tip arrives, and then will oscillate near the static value. A simple explicit expression of the dynamic overshoot for stress-intensity factors is derived as a function of the location for applied loadings, the crack length and material constants.

Keywords: finite cracks; anisotropic materials; dynamic fracture; dynamic stress-intensity factors; transient waves; dynamic overshoot

\section{Introduction}

Since the late nineteenth century, the study of stress concentration of an elastic body with cracks, cavities, inclusions or other types of discontinuities has been the major 
subject of elastostatics. The question of whether the static stress-intensity factors are applicable to dynamic response was not answered until the phenomenon of scattering of elastic waves was investigated in the late 1950s. In general, the dynamic stressintensity factors are greater than the corresponding static values so that the inertia effects of structures cannot be neglected in many dynamic designs, such as aircrafts, vessels and nuclear plants, etc. Hence, the investigation of dynamic fracture problems has become more important and has received much attention by many researchers. The major reference on the dynamic fracture mechanics is the extensive monograph by Freund (1990). Most of the analysis typically provided the time history of the dynamic stress-intensity factor.

Scattering of elastic waves by cracks has attracted attention over the years for its importance towards the non-destructive evaluation of materials and the dynamic fracture analysis of materials. The interaction of a stress wave with a crack is a complicated problem and the analysis is mainly restricted to relatively simple problems. Most of the works for dynamic fracture analysis, however, have been directed towards the solutions of problems without any characteristic length. The complete solutions of this kind of problems can be obtained by integral transform methods in conjunction with direct application of the Wiener-Hopf technique (Noble 1958) and the Cagniard-de Hoop method (de Hoop 1958) of Laplace inversion. If the cracked problem has a characteristic length or the loading condition is unsymmetrical, then the same procedure using integral transform methods is difficult to apply. The problem of an unbounded isotropic elastic solid containing a semi-infinite crack subjected to a pair of concentrated point loadings on the crack faces has been studied by Freund (1974). A straightforward application of the Wiener-Hopf method was not successful and he proposed a fundamental solution arising from an edge dislocation climbing along the line ahead of the crack tip with a constant speed to overcome the difficulties of the case with a characteristic length. The solution can be constructed by taking integration over a climbing edge dislocation of different moving velocity. Basing the procedure on this method, Brock (1982, 1984), Brock et al. (1985) and Ma \& Hou (1990, 1991) have analysed a series of problems of an unbounded medium containing a semi-infinite crack subjected to impact loadings. The limitation of the above-mentioned problems is that the incident field must be represented as a function of $f(t / x)$, say self-similar. For problems of a non-infinite domain or with complicated loading conditions, the solutions cannot be obtained by using this method. A powerful and efficient methodology based on superimposing a fundamental solution in the Laplace transform domain was proposed by Tsai \& Ma (1992). Exact transient closed-form solutions of stresses and dynamic stress-intensity factors for a stationary semi-infinite crack subjected to a suddenly applied dynamic body force in an unbounded medium have been obtained by Tasi \& Ma (1992) for the in-plane case. This fundamental solution and superposition methodology have also been successfully applied by Ma \& Chen (1994) to solve the more complicated problem of a half-plane containing a stationary semi-infinite inclined crack for antiplane deformation. This superposition methodology was generalized and applied to analyse the propagating crack interacting with boundaries for in-plane deformation by Tsai \& Ma $(1997 a, b)$, but only the effects of first few reflected waves from the boundaries were taken into account.

Many researchers have already studied finite-crack problems for isotropic materials. The stress-intensity factor of a finite crack subjected to time-harmonic, hori-

Proc. R. Soc. A (2005) 
zontally polarized plane wave has been obtained by Loeber \& Sih (1968) and Sih \& Loeber (1969). Thau \& Lu (1971), following the work of Kostrov (1964) and Flitman (1963), treated the analogous transient problem of diffraction of an arbitrary plane dilatational wave by a finite crack in an infinite elastic solid. Their results are exact only for the time-interval during which the dilatational wave travelled the length of the crack twice. Sih \& Embley (1972) have studied the near-field solution for the problem of a finite crack under transient in-plane loading. They reduced the mixed boundary-value problem to a standard Fredholm integral equation and subsequently inverted the Laplace transform of the stress components by a combination of numerical means and an application of the Cagniard inversion technique. Most of the investigators, however, have either solved problems of a finite crack valid for a short time, or finally used a numerical technique to obtain the solutions in the physical domain. Because of the mathematical difficulties, the transient closed-form analytical solution for the problem of a finite crack was not attempted until Ing \& Ma $(1996,1997)$ proposed useful fundamental solutions to overcome this difficulty. Ing \& Ma $(1996,1997)$ solved the problem of a finite crack subjected to a dynamic anti-plane point loading and a horizontally polarized shear wave in isotropic solids, but only dynamic stress-intensity factors were obtained in closed forms.

Recently, due to the fast development in material science, anisotropic materials have been widely used for engineering applications. Material anisotropy may occur in many solids such as composites and piezoelectric ceramics. Recent developments in anisotropic elasticity were reviewed by Ting (2000). Ting (1996) pointed out that there have been several new developments in the theory and applications of anisotropic elasticity. However, few results for dynamic fracture analysis of anisotropic solids can be found in the literature. Rizza \& Nair (1999) have used a numerical method to solve problems involving non-axisymmetric dynamic impact loading of a penny-shaped crack in a transversely isotropic medium. The threedimensional problem for a transversely isotropic crack subjected to suddenly applied concentrated point forces has been analysed by Zhao (2001). Sarkar et al. (1995) have analysed the dynamic response of three co-planar Griffith cracks in an orthotropic medium. They used the Fourier transform and finite Hilbert transform techniques to obtain approximate values of the crack-opening displacements and stress-intensity factors. The time-harmonic problem of two parallel cracks in an infinite orthotropic plane was studied by Itou \& Haliding (1997). They calculated the dynamic stressintensity factors numerically for composite materials. Das \& Patra $(1996,1998)$ have investigated the plane-strain problem of dynamic stress-intensity factors for a moving Griffith crack situated at the interface of two dissimilar orthotropic fixed layers and half-planes. The authors solved the reduced singular integral equations by using Jacobi's polynomials. Liu \& Achenbach $(1994,1995)$ have extended the strip-element method to investigate wave scattering by cracks in anisotropic laminated plates. Liu et al. (1999) and $\mathrm{Xi}$ et al. (2000) have studied numerically the wave scattering by a crack in a composite laminate submerged in a fluid and in a fluid-filled composite cylindrical shell. Shen \& Kuang (1998) investigated the problem of wave scattering from an interface crack in laminated anisotropic media. In their study, the wave fields were obtained numerically. Pramanik et al. (1999) analysed the high-frequency scattering of a finite interface crack subjected to time-harmonic anti-plane loading in dissimilar anisotropic materials and derived the stress-intensity factor at the crack tip. Ma \& Liao (1999) studied the transient full-field response of a semi-infinite inter- 
face crack lying between dissimilar anisotropic media subjected to a dynamic body force. Zhang (2000) applied the time-domain traction boundary integral equation (BIE) to obtain the dynamic stress-intensity factor of a finite crack in anisotropic solids under anti-plane deformation.

Finite cracks in orthotropic materials under dynamic loading have been investigated by Kassir \& Bandyopadhyay (1983), Shindo \& Nozaki (1991) and RubioGonzalez \& Mason $(1999,2001)$ using the integral transform method. This method leads to a Fredholm integral equation rather than a Wiener-Hopf equation on the Laplace transform domain. They solved the Fredholm integral equation in the Laplace transform domain numerically, and the dynamic stress-intensity factor was obtained by numerical Laplace inversion. Rubio-Gonzalez \& Mason (2000) obtained analytical closed-form solutions for dynamic stress-intensity factors of a semi-infinite crack under uniform crack face loading in orthotropic materials. Most of the previous studies on elastodynamic crack analysis of anisotropic solids were limited to transversely isotropic or orthotropic solids.

Because of the mathematical difficulties, an explicit analytical solution for the problem of a finite crack subjected to dynamic point loading in anisotropic solids has not been obtained. It is important to develop a mathematical method to construct exact full-field closed-form solutions for finite cracks in anisotropic materials. In this study, the theoretical transient analysis is performed for a finite crack in an unbounded anisotropic medium subjected to an anti-plane concentrated dynamic loading as shown in figure 1. The total wave field is due to the effect of this concentrated loading and the scattering of the incident wave by two crack tips. Investigations on the problems for anisotropic materials are tedious due to the presence of many material constants. It is desirable to reduce the dependence on material constants in advance of the analysis of a given problem. A linear coordinate transformation is introduced in this study to simplify the problem. Based on this transformation, the original problem of anisotropic materials with a finite crack is converted to an equivalent isotopic problem with a similar geometrical configuration. In analysing this problem, the interaction of waves with two crack tips must be taken into account and it is impossible to solve this complicated problem by using the standard integral transform method. Two useful fundamental solutions are proposed to overcome these difficulties. In this study, the exact dynamic full-field solutions of shear stresses and displacement and stress-intensity factor are derived and expressed in closed forms. Based on the analytical solutions, the transient responses of shear stresses and stressintensity factor are discussed in detail using numerical calculations. In some classes of dynamic problems, the ability to find a static field may hinge on waiting for the wavefronts to pass and the transient effect to die away. One of the main objectives in this study is to investigate the characteristic time after which the transient solution would approach the correspondent static value. The transient behaviour of shear stress is analysed, and it is found that the transient effect can be neglected after the first few waves have passed the field point. The analytical solutions show that the dynamic stress-intensity factor will reach a maximum value when the incident wave reaches the crack tip, remains constant before the diffracted wave generated from the other crack tip arrives, and then decreases and oscillates near the static value. The explicit formulation of the dynamic overshoot of the stress-intensity factor is presented in a simple expression which provides important information for dynamic fracture analysis.

Proc. R. Soc. A (2005) 


\section{A linear coordinate transformation and fundamental solutions}

For the anti-plane shear deformation, the displacement is parallel to the axial coordinate that is normal to the plane and is dependent only on the coordinates in the plane. Such a deformation field characterized by a single axial displacement can be regarded as complementary to that of plane-strain deformation. The anti-plane problem plays a useful role as a pilot problem that reveals simpler aspects of elasticity solutions. Analysis of anisotropic elasticity problems is often tedious due to the presence of many elastic constants. It is desirable to reduce the dependence on elastic constants through theoretical considerations in advance of the analysis of a given boundary-value problem. The anti-plane anisotropic problem can be converted to a corresponding isotropic problem by properly changing the geometry of the original configuration and the tractions on the boundary. In other words, the anisotropic antiplane problem can be simplified to an isotropic problem with the aid of a suitable coordinate transformation. By using a linear coordinate transformation, the complete full-field static solutions of anisotropic multilayered media subjected to concentrated shear forces and screw dislocations in an arbitrary layer were obtained by Lin \& Ma (2000).

The anti-plane problem is usually a precursor to the more practically useful inplane problems. The problem of anisotropic elasticity for in-plane deformations contains six elastic constants in a single material phase. Significant reduction of the problem was achieved by the Cherkaev-Lurie-Milton (CLM) (Cherkaev et al. 1992) shift, which effectively eliminated two elastic constants in most cases. Dundurs \& Markenscoff (1993) generalized the uniform CLM shift to include a linear alternation of the elastic compliance. The well-known Dundurs constants for isotropic elastic biomaterials have been extended to anisotropic biomaterials by Ting (1995). Recently, Yang \& Ma (1998) combined the CLM shift and an orthotropic transformation to further reduce dependence on elastic constants for in-plane anisotropic problems.

In this section, a linear coordinate transformation and two fundamental problems are proposed to solve the aforementioned problem of a finite crack in an anisotropic material subjected to dynamic concentrated loadings. The solutions for an exponentially distributed traction applied on the crack faces and an exponentially distributed displacement along the crack tip line in the Laplace transform domain will be referred to as the fundamental solutions. The diffracted waves generated from two crack tips can be successfully constructed by superimposing the fundamental solutions in the Laplace transform domain.

\section{(a) The linear coordinate transformation}

Consider a fundamental problem of an anti-plane deformation for a semi-infinite crack contained in an anisotropic unbounded medium. For the absence of body force, the two-dimensional anti-plane wave motion of a homogeneous anisotropic solid in terms of the displacement is governed by

$$
C_{55} \frac{\partial^{2} w(x, y, t)}{\partial x^{2}}+2 C_{45} \frac{\partial^{2} w(x, y, t)}{\partial x \partial y}+C_{44} \frac{\partial^{2} w(x, y, t)}{\partial y^{2}}=\rho^{2} \frac{\partial^{2} w(x, y, t)}{\partial t^{2}},
$$

where $w(x, y, t)$ is the out-of-plane displacement in the $z$-direction, $C_{i j}(i, j=4,5)$ are elastic moduli and $\rho$ is the mass density of the anisotropic material. The $(x, y)$ plane has been assumed to coincide with one of the planes of material symmetry, so

Proc. R. Soc. A (2005) 
that in-plane and anti-plane deformations are uncoupled. The relevant shear-stress components are

$$
\begin{aligned}
& \tau_{y z}(x, y, t)=C_{44} \frac{\partial w(x, y, t)}{\partial y}+C_{45} \frac{\partial w(x, y, t)}{\partial x}, \\
& \tau_{x z}(x, y, t)=C_{45} \frac{\partial w(x, y, t)}{\partial y}+C_{55} \frac{\partial w(x, y, t)}{\partial x} .
\end{aligned}
$$

Introduce a linear coordinate transformation (Ma 1996; Lin \& Ma 2000)

$$
\begin{aligned}
X & =x-\frac{C_{45}}{C_{44}} y, \\
Y & =\frac{C_{e}}{C_{44}} y, \\
Z & =z,
\end{aligned}
$$

where

$$
C_{e}=\sqrt{C_{44} C_{55}-C_{45}^{2}}
$$

Assume that $C_{44}, C_{45}$ and $\sqrt{C_{44} C_{55}-C_{45}^{2}}$ are all positive. The transformation given by (2.4)-(2.6) reduces (2.1) to the standard wave equation for the isotropic solid in the $(X, Y)$-coordinate system as

$$
\frac{\partial^{2} W(X, Y, t)}{\partial X^{2}}+\frac{\partial^{2} W(X, Y, t)}{\partial Y^{2}}=b^{2} \frac{\partial^{2} W(X, Y, t)}{\partial t^{2}},
$$

where $W(X, Y, t)$ is the displacement in the $Z$-direction and

$$
\begin{gathered}
W(X, Y, t)=w(x, y, t), \\
b=\frac{\sqrt{C_{44} \rho}}{C_{e}} .
\end{gathered}
$$

It is easy to verify from (2.2) and (2.3) that the relevant stress components in the anisotropic solid are related to those in the corresponding isotropic solid by

$$
\begin{aligned}
\tau_{Y Z}(X, Y, t) & =C_{e} \frac{\partial W(X, Y, t)}{\partial Y}, \\
\tau_{X Z}(X, Y, t) & =C_{e} \frac{\partial W(X, Y, t)}{\partial X}, \\
\tau_{y z}(x, y, t) & =\tau_{Y Z}(X, Y, t), \\
\tau_{x z}(x, y, t) & =\frac{C_{45}}{C_{44}} \tau_{Y Z}(X, Y, t)+\frac{C_{e}}{C_{44}} \tau_{X Z}(X, Y, t) .
\end{aligned}
$$

From (2.8), (2.11) and (2.12), it is noted that the original anisotropic problem is converted into an equivalent isotropic problem by setting $C_{e}=\mu$ (shear modulus). From the relationship of displacement and shear stresses for an anisotropic solid and the correspondent isotropic solid expressed in (2.9), (2.13) and (2.14), one can see that it is possible to obtain the solution for an anisotropic problem from a corresponding result of the isotropic problem. 


\section{(b) The fundamental solution of distributed loads on} anisotropic semi-infinite crack faces

Consider an anisotropic material in an unbounded medium containing a semiinfinite crack which lies on the negative $x$-axis. An anti-plane exponentially distributed traction in the Laplace transform domain is applied on the upper and lower crack faces. The boundary conditions on crack surfaces expressed in the Laplace transform domain for this fundamental problem are represented as follows

$$
\begin{aligned}
\bar{\tau}_{y z}(x, 0, s) & =\mathrm{e}^{s \eta x}, & -\infty & <x<0, \\
\bar{w}(x, 0, s) & =0, & 0 & <x<\infty,
\end{aligned}
$$

where $s$ is the Laplace transform parameter and $\eta$ is a constant. The overbar is used to denote the transform on time $t$. By using (2.4)-(2.6), (2.9) and (2.13), the boundary conditions equations (2.15) and (2.16) are rewritten as

$$
\begin{aligned}
\bar{\tau}_{Y Z}(X, 0, s) & =\mathrm{e}^{s \eta X}, & -\infty & <X<0, \\
\bar{W}(X, 0, s) & =0, & 0 & <X<\infty .
\end{aligned}
$$

The fundamental problem for the governing equation (2.8) subjected to boundary conditions (2.17) and (2.18) can be solved by the application of standard integral transform methods. The one-sided Laplace transform with respect to time and the two-sided Laplace transform with respect to $X$ are defined by (Achenbach 1973)

$$
\begin{aligned}
& \bar{f}(X, Y, s)=\int_{0}^{\infty} f(X, Y, t) \mathrm{e}^{-s t} \mathrm{~d} t, \\
& \bar{f}^{*}(\lambda, Y, s)=\int_{-\infty}^{\infty} \bar{f}(X, Y, s) \mathrm{e}^{-s \lambda X} \mathrm{~d} X .
\end{aligned}
$$

Applying the one-sided Laplace transform over time, and the two-sided Laplace transform over $x$ under the restriction of $\operatorname{Re}(\eta)>\operatorname{Re}(\lambda)$, and the Wiener-Hopf technique is finally implemented. The solutions of shear stresses and displacement in the Laplace transform domain can be expressed as

$$
\begin{aligned}
\bar{\tau}_{Y Z}(X, Y, s) & =\frac{1}{2 \pi \mathrm{i}} \int_{\Gamma_{\lambda}} \frac{\alpha_{+}(\lambda) \exp \{-s[\alpha(\lambda)|Y|-\lambda X]\}}{\alpha_{+}(\eta)(\eta-\lambda)} \mathrm{d} \lambda, \\
\bar{\tau}_{X Z}(X, Y, s) & =\frac{-\operatorname{sgn}(Y)}{2 \pi \mathrm{i}} \int_{\Gamma_{\lambda}} \frac{\lambda \exp \{-s[\alpha(\lambda)|Y|-\lambda X]\}}{\alpha_{+}(\eta)(\eta-\lambda) \alpha_{-}(\lambda)} \mathrm{d} \lambda, \\
\bar{W}(X, Y, s) & =\frac{-\operatorname{sgn}(Y)}{2 \pi \mathrm{i}} \int_{\Gamma_{\lambda}} \frac{\exp \{-s[\alpha(\lambda)|Y|-\lambda X]\}}{C_{e} s \alpha_{+}(\eta)(\eta-\lambda) \alpha_{-}(\lambda)} \mathrm{d} \lambda,
\end{aligned}
$$

where $\Gamma_{\lambda}$ is the path of integration in the complex $\lambda$-plane and

$$
\begin{gathered}
\operatorname{sgn}(Y)= \begin{cases}1, & Y \geqslant 0^{+}, \\
-1, & Y \leqslant 0^{-},\end{cases} \\
\alpha_{+}(\lambda)=\sqrt{b+\lambda,} \quad \alpha_{-}(\lambda)=\sqrt{b-\lambda}, \\
\alpha(\lambda)=\alpha_{+}(\lambda) \alpha_{-}(\lambda) .
\end{gathered}
$$

Proc. R. Soc. A (2005) 
The corresponding result for the dynamic stress-intensity factor in the Laplace transform domain is

$$
\bar{K}(s)=\lim _{x \rightarrow 0^{+}} \sqrt{2 \pi X} \bar{\tau}_{Y Z}(x, 0, s)=-\frac{\sqrt{2}}{\sqrt{s} \alpha_{+}(\eta)} .
$$

\section{(c) The fundamental solution of distributed displacement ahead of the anisotropic semi-infinite crack tip}

Consider a semi-infinite crack contained in an unbounded anisotropic medium. An exponentially distributed screw dislocation ahead of the crack tip yields the following boundary conditions in the Laplace transform domain:

$$
\begin{array}{lrlrl}
\bar{w}\left(x, 0^{+}, s\right) & =\mathrm{e}^{s \eta x}, & 0 & <x<\infty, \\
\bar{w}\left(x, 0^{-}, s\right) & =-\mathrm{e}^{s \eta x}, & 0 & <x<\infty, \\
\bar{\tau}_{y z}(x, 0, s) & =0, & -\infty & <x<0 .
\end{array}
$$

We then follow a similar procedure to one mentioned previously. The solutions for stresses and displacement expressed in the Laplace transform domain can be obtained, and the results are

$$
\begin{aligned}
\bar{\tau}_{Y Z}(X, Y, s) & =\frac{1}{2 \pi \mathrm{i}} \int_{\Gamma_{\lambda}} \frac{C_{e} \alpha_{-}(\eta) \alpha_{+}(\lambda) \exp \{-s[\alpha(\lambda)|Y|(-\lambda X)]\}}{(\eta-\lambda)} \mathrm{d} \lambda, \\
\bar{\tau}_{X Z}(X, Y, s) & =\frac{-\operatorname{sgn}(Y)}{2 \pi \mathrm{i}} \int_{\Gamma_{\lambda}} \frac{C_{e} s \alpha_{-}(\eta) \lambda \exp \{-s[\alpha(\lambda)|Y|-\lambda X]\}}{(\eta-\lambda) \alpha_{-}(\lambda)} \mathrm{d} \lambda, \\
\bar{W}(X, Y, s) & =\frac{-\operatorname{sgn}(Y)}{2 \pi \mathrm{i}} \int_{\Gamma_{\lambda}} \frac{\alpha_{-}(\eta) \exp \{-s[\alpha(\lambda)|Y|-\lambda X]\}}{(\eta-\lambda) \alpha_{-}(\lambda)} \mathrm{d} \lambda .
\end{aligned}
$$

The corresponding result for the stress-intensity factor expressed in the Laplace transform domain is

$$
\bar{K}(s)=-C_{e} \sqrt{2 s} \alpha_{-}(\eta) .
$$

\section{Exact transient full-field solutions for a finite crack}

The problem to be considered here is an infinite medium containing a finite crack of length $l$ as shown in figure 1. The infinite cracked body is made of an anisotropic, homogeneous, and linearly elastic material. The origins of two coordinate systems $(x, y)$ and $\left(x^{\prime}, y^{\prime}\right)$ are located at crack tips $\mathrm{A}$ and $\mathrm{B}$, respectively. For time $t<$ 0 , the medium is stress free and at rest. At time $t=0$, an anti-plane dynamic concentrated loading with magnitude $2 P$ is applied suddenly at $x=h_{1}, y=h_{2}$ (where $h_{2}>0$ ). The time dependence of the concentrated loading is represented by the Heaviside step function $\mathcal{H}(t)$. The interaction of an incident wave with the finite crack induces scattered waves. The total wave field is the sum of the incident wave and the scattered wave field. After applying the concentrated impact loading, the incident stress wave with an elliptic shape of wavefront generated from the loading point will propagate towards the crack, a reflected wave will be generated from the crack face and two diffracted waves will be induced from two crack tips. When the first diffracted wave generated from crack tip B arrives at crack tip A after some later 


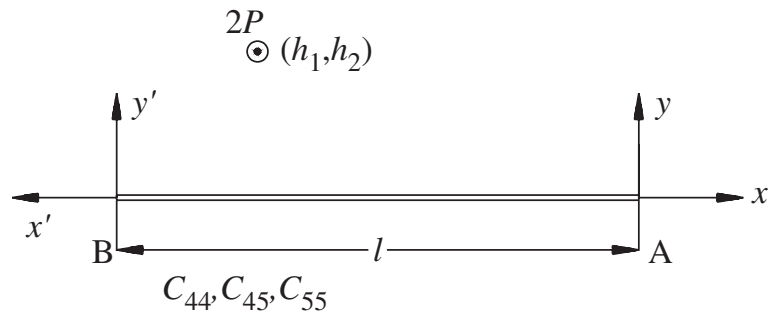

Figure 1. The loading and coordinate systems of a finite crack in an anisotropic medium.

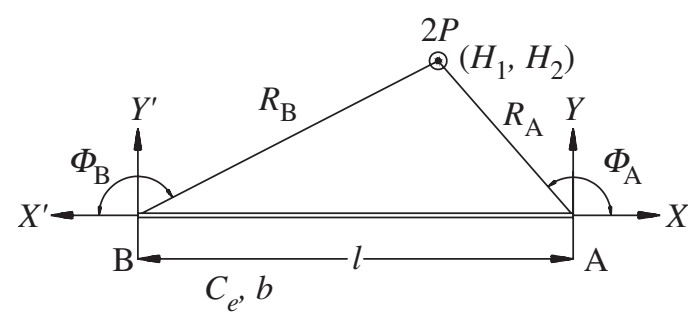

Figure 2. The loading and coordinate systems of figure 1 after using the linear coordinate transformation.

time, a second diffracted wave will be induced from crack tip A. Then this second diffracted wave will reach at crack tip B and a third diffracted wave is generated. Hence the diffracted waves will scatter back and forth between the crack tips A and B. Follow a similar procedure continuously, the complete full-fields solutions that account for the contributions of all the diffracted waves induced from two crack tips can be derived. In analysing this problem, the multiple diffractions of stress waves by the finite crack must be taken into account. This is the most difficult part of analysing the problem, and fundamental solutions provided in the previous section will be very useful in overcoming this difficulty. Furthermore, a linear coordinate transformation and an effective superposition scheme are also used to solve the problem. Exact transient full-field solutions of displacement and shear stresses that account for the contributions of all the diffracted waves are presented in this section.

The anti-plane concentrated impact loading applied at $\left(h_{1}, h_{2}\right)$ in the unbounded medium can be described as a jump condition for the shear stress as follows:

$$
\tau_{y z}\left(x, h_{2}^{+}, t\right)-\tau_{y z}\left(x, h_{2}^{-}, t\right)=2 P \delta\left(x-h_{1}\right) \mathcal{H}(t),
$$

where $\mathcal{H}(\cdot)$ and $\delta(\cdot)$ are the Heaviside step and Dirac delta functions, respectively. Introduce a linear coordinate transformation as indicated in (2.4)-(2.6). Then the configuration and coordinate systems as shown in figure 1 for anisotropic materials are changed to those presented in figure 2 for isotropic materials. It is interesting to see from figure 2 that the crack length is $l$, but the location of the concentrated loading has shifted to $(X, Y)=\left(H_{1}, H_{2}\right)$ with the same magnitude $2 P$, where

$$
H_{1}=h_{1}-\frac{C_{45}}{C_{44}} h_{2}, \quad H_{2}=\frac{C_{e}}{C_{44}} h_{2} .
$$

The jump condition in (3.1) can be rewritten as

$$
\tau_{Y Z}\left(X, H_{2}^{+}, t\right)-\tau_{Y Z}\left(X, H_{2}^{-}, t\right)=2 P \delta\left(X-H_{1}\right) \mathcal{H}(t) .
$$

Proc. R. Soc. A (2005) 
Before the incident wave generated by the applied loading interacts with the finite crack, the problem can be treated as wave propagation in an unbounded medium. From (3.2), the incident stress field expressed in the Laplace transform domain is given by

$$
\bar{\tau}_{Y Z}^{\mathrm{i}}(X, Y, s)=\frac{-\operatorname{sgn}\left(Y-H_{2}\right)}{2 \pi \mathrm{i}} \int_{\Gamma_{\lambda}} P \exp \left\{-s \alpha(\lambda)\left|Y-H_{2}\right|+s \lambda\left(X-H_{1}\right)\right\} \mathrm{d} \lambda .
$$

From (2.11) and (2.12), we can obtain the corespondent displacement and shearstress component as follows:

$$
\begin{aligned}
& \bar{\tau}_{X Z}^{\mathrm{i}}(X, Y, s)=\frac{1}{2 \pi \mathrm{i}} \int_{\Gamma_{\lambda}} \frac{P \lambda}{\alpha(\lambda)} \exp \left\{-s \alpha(\lambda)\left|Y-H_{2}\right|+s \lambda\left(X-H_{1}\right)\right\} \mathrm{d} \lambda, \\
& \bar{W}^{\mathrm{i}}(X, Y, s)=\frac{1}{2 \pi \mathrm{i}} \int_{\Gamma_{\lambda}} \frac{P}{C_{e} s \alpha(\lambda)} \exp \left\{-s \alpha(\lambda)\left|Y-H_{2}\right|+s \lambda\left(X-H_{1}\right)\right\} \mathrm{d} \lambda .
\end{aligned}
$$

By using the Cagniard-de Hoop method of Laplace inversion to (3.3)-(3.5), the incident transient fields in time domain are obtained as

$$
\begin{aligned}
\tau_{Y Z}^{\mathrm{i}}(X, Y, t) & =\frac{-\operatorname{sgn}\left(Y-H_{2}\right) P t \sin \left(\Phi_{i}\right)}{\pi R_{i}\left(t^{2}-b^{2} R_{i}^{2}\right)^{1 / 2}} \mathcal{H}\left(t-b R_{i}\right), \\
\tau_{X Z}^{\mathrm{i}}(X, Y, t) & =\frac{-P t \cos \left(\Phi_{i}\right)}{\pi R_{i}\left(t^{2}-b^{2} R_{i}^{2}\right)^{1 / 2}} \mathcal{H}\left(t-b R_{i}\right), \\
W^{\mathrm{i}}(X, Y, t) & =\frac{P}{\pi C_{e}} \ln \left[\frac{t}{b R_{i}}+\left(\frac{t}{b R_{i}}\right)^{2}-1\right] \mathcal{H}\left(t-b R_{i}\right),
\end{aligned}
$$

where

$$
R_{i}=\left[\left(X-H_{1}\right)^{2}+\left(Y-H_{2}\right)^{2}\right]^{1 / 2}, \quad \Phi_{i}=\arccos \left(\frac{X-H_{1}}{R_{i}}\right)
$$

Without loss of generality, we assume that the incident wave will arrive at crack tip A first. Before the incident wave is diffracted from crack tip B, the stress field is precisely the same as that derived for a semi-infinite crack lying in the plane $Y=0$ and $-\infty<X<0$ and is subjected to the same loading. From (3.3), the incident stress field $\bar{\tau}_{y z}^{\mathrm{i}}(x, 0, s)$ at $y=0$ generated by the applied dynamic loading is

$$
\bar{\tau}_{Y Z}^{\mathrm{i}}(X, 0, s)=\frac{1}{2 \pi \mathrm{i}} \int_{\Gamma_{\lambda}} P \exp \left\{-s\left[\alpha(\lambda) H_{2}+\lambda H_{1}\right]+s \lambda X\right\} \mathrm{d} \lambda .
$$

The applied traction on the crack face, in order to eliminate the incident wave as indicated in (3.9), has the functional form $\mathrm{e}^{s \lambda x}$. Since the solutions for applying traction $\mathrm{e}^{s \eta x}$ on crack faces were obtained in $\S 2$, the reflected and diffracted fields can be constructed by superimposing the incident wave traction that is equal and opposite to (3.9). By using (2.21)-(2.23) and (3.9), the solutions for the reflected wave and the first diffracted wave induced by crack tip A in the Laplace transform 
domain can be expressed as follows:

$$
\begin{aligned}
& \bar{W}^{\mathrm{A}}(X, Y, s)=\frac{-1}{2 \pi \mathrm{i}} \int_{\Gamma_{\eta_{1}}} P \exp \left\{-s\left[\alpha\left(\eta_{1}\right) h_{2}+\eta_{1} h_{1}\right]\right\} \\
& \times\left\{\frac{1}{2 \pi \mathrm{i}} \int_{\Gamma_{\eta_{2}}} \frac{-\operatorname{sgn}(Y) \exp \left\{-s\left[\alpha\left(\eta_{2}\right)|Y|-\eta_{2} X\right]\right\}}{C_{e} s \alpha_{+}\left(\eta_{1}\right)\left(\eta_{1}-\eta_{2}\right) \alpha_{-}\left(\eta_{2}\right)} \mathrm{d} \eta_{2}\right\} \mathrm{d} \eta_{1}
\end{aligned}
$$

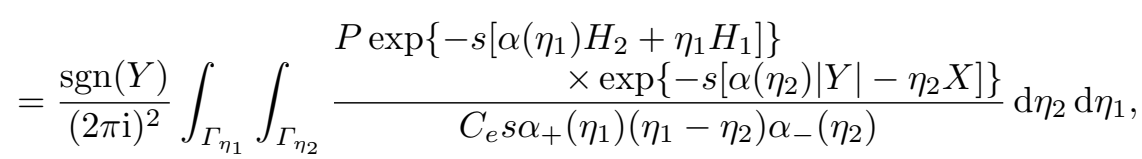

$$
\begin{aligned}
& \bar{\tau}_{Y Z}^{\mathrm{A}}(X, Y, s)=\frac{-1}{(2 \pi \mathrm{i})^{2}} \int_{\Gamma_{\eta_{1}}} \int_{\Gamma_{\eta_{2}}} \frac{\begin{array}{c}
P \alpha_{+}\left(\eta_{2}\right) \exp \left\{-s\left[\alpha\left(\eta_{1}\right) H_{2}+\eta_{1} H_{1}\right]\right\} \\
\times \exp \left\{-s\left[\alpha\left(\eta_{2}\right)|Y|-s \eta_{2} X\right]\right\}
\end{array}}{\alpha_{+}\left(\eta_{1}\right)\left(\eta_{1}-\eta_{2}\right)} \mathrm{d} \eta_{2} \mathrm{~d} \eta_{1}
\end{aligned}
$$

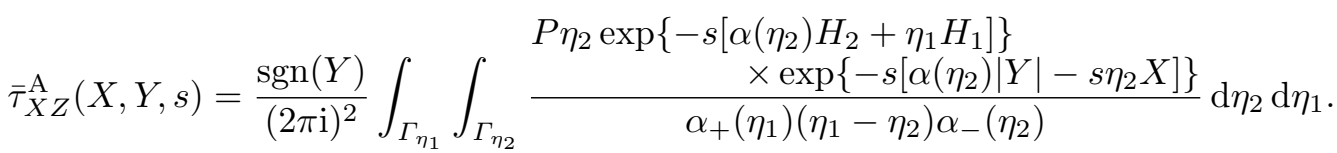

Applying the inverse Laplace transform to (3.10)-(3.12) yields the displacement and shear stresses:

$$
\begin{aligned}
& W^{\mathrm{A}}(X, Y, t) \\
& =W^{\mathrm{A}_{1}}(X, Y, t)+W^{r}(X, Y, t) \\
& =\frac{-\operatorname{sgn}(Y) P}{2 \pi^{2} C_{e}} \int_{b R+b R_{\mathrm{A}}}^{t} \int_{b R_{\mathrm{A}}}^{\tau-b R} \operatorname{Re}\left[\frac{\left(\partial \eta_{1}^{+} / \partial t_{1}\right)\left(\partial \eta_{2}^{+} / \partial t_{2}\right)}{\alpha_{+}\left(\eta_{1}^{+}\right)\left(\eta_{1}^{+}-\eta_{2}^{+}\right) \alpha_{-}\left(\eta_{2}^{+}\right)}\right. \\
& \left.-\frac{\left(\partial \eta_{1}^{-} / \partial t_{1}\right)\left(\partial \eta_{2}^{+} / \partial t_{2}\right)}{\alpha_{+}\left(\eta_{1}^{-}\right)\left(\eta_{1}^{-}-\eta_{2}^{+}\right) \alpha_{-}\left(\eta_{2}^{+}\right)}\right]_{t=\tau} \mathrm{d} t_{1} \mathrm{~d} \tau \\
& +\frac{\operatorname{sgn}(Y) P}{\pi C_{e}} \ln \left[\frac{t}{b R_{r}}+\left(\frac{t}{b R_{r}}\right)^{2}-1\right] \mathcal{H}\left(t-b R_{r}\right) \mathcal{H}\left(\operatorname{sgn}(Y) \Phi-\pi+\Phi_{\mathrm{A}}\right), \\
& \tau_{Y Z}^{\mathrm{A}}(X, Y, t)=\tau_{Y Z}^{\mathrm{A}_{1}}(X, Y, t)+\tau_{Y Z}^{r}(X, Y, t) \\
& =\frac{P}{2 \pi^{2}} \int_{b R_{\mathrm{A}}}^{t-b R} \operatorname{Re}\left[\frac{\alpha_{+}\left(\eta_{2}^{+}\right)\left(\partial \eta_{1}^{+} / \partial t_{1}\right)\left(\partial \eta_{2}^{+} / \partial t_{2}\right)}{\alpha_{+}\left(\eta_{1}^{+}\right)\left(\eta_{1}^{+}-\eta_{2}^{+}\right)}\right. \\
& \left.-\frac{\alpha_{+}\left(\eta_{2}^{+}\right)\left(\partial \eta_{1}^{-} / \partial t_{1}\right)\left(\partial \eta_{2}^{+} / \partial t_{2}\right)}{\alpha_{+}\left(\eta_{1}^{-}\right)\left(\eta_{1}^{-}-\eta_{2}^{+}\right)}\right] \mathrm{d} t_{1} \\
& -\frac{P t \sin \left(\Phi_{r}\right)}{\pi R_{r}\left(t^{2}-b^{2} R_{r}^{2}\right)^{1 / 2}} \mathcal{H}\left(t-b R_{r}\right) \mathcal{H}\left(\operatorname{sgn}(Y) \Phi-\pi+\Phi_{\mathrm{A}}\right),
\end{aligned}
$$

Proc. R. Soc. A (2005) 


$$
\begin{aligned}
\tau_{X Z}^{\mathrm{A}}(X, Y, t)= & \tau_{X Z}^{\mathrm{A}_{1}}(X, Y, t)+\tau_{X Z}^{r}(X, Y, t) \\
=\frac{-\operatorname{sgn}(Y) P}{2 \pi^{2}} \int_{b R_{\mathrm{A}}}^{t-b R} \operatorname{Re}\left[\eta_{2}^{+} \frac{\left(\partial \eta_{1}^{+} / \partial t_{1}\right)\left(\partial \eta_{2}^{+} / \partial t_{2}\right)}{\alpha_{+}\left(\eta_{1}^{+}\right)\left(\eta_{1}^{+}-\eta_{2}^{+}\right) \alpha_{-}\left(\eta_{2}^{+}\right)}\right. & \left.\quad-\frac{\eta_{2}^{+}\left(\partial \eta_{1}^{-} / \partial t_{1}\right)\left(\partial \eta_{2}^{+} / \partial t_{2}\right)}{\alpha_{+}\left(\eta_{1}^{-}\right)\left(\eta_{1}^{-}-\eta_{2}^{+}\right) \alpha_{-}\left(\eta_{2}^{+}\right)}\right] \mathrm{d} t_{1} \\
& \quad-\frac{\operatorname{sgn}(Y) P t \cos \left(\Phi_{r}\right)}{\pi R_{r}\left(t^{2}-b^{2} R_{r}^{2}\right)^{1 / 2}} \mathcal{H}\left(t-b R_{r}\right) \mathcal{H}\left(\operatorname{sgn}(Y) \Phi-\pi+\Phi_{\mathrm{A}}\right),
\end{aligned}
$$

where

$$
\begin{gathered}
\eta_{1}^{ \pm}=\frac{t_{1} \cos \Phi_{\mathrm{A}}}{R_{\mathrm{A}}} \pm \mathrm{i} \frac{\sin \Phi_{\mathrm{A}}}{R_{\mathrm{A}}}\left(t_{1}^{2}-b^{2} R_{\mathrm{A}}^{2}\right)^{1 / 2}, \quad \eta_{2}^{ \pm}=\frac{-t_{2} \cos \Phi}{R} \pm \mathrm{i} \frac{\sin \Phi}{R}\left(t_{2}^{2}-b^{2} R^{2}\right)^{1 / 2}, \\
R_{\mathrm{A}}=\left(H_{1}^{2}+H_{2}^{2}\right)^{1 / 2}, \quad \Phi_{\mathrm{A}}=\arccos \left(\frac{H_{1}}{R_{\mathrm{A}}}\right), \quad R=\left(X^{2}+Y^{2}\right)^{1 / 2}, \\
\Phi=\operatorname{sgn}(Y) \arccos \left(\frac{X}{R}\right), \quad R_{r}=\left[\left(X-H_{1}\right)^{2}+\left(|Y|+H_{2}\right)^{2}\right]^{1 / 2}, \\
\Phi_{r}=\arccos \left(\frac{X-H_{1}}{R_{r}}\right), \quad t_{1}+t_{2}=t .
\end{gathered}
$$

The results expressed in (3.13)-(3.15) are the exact transient solutions for a semiinfinite crack in an infinite medium subjected to point-impact loading. The first and second terms in (3.13)-(3.15) represent the diffracted and reflected waves, respectively.

In order to obtain the first diffracted wave induced from crack tip B, the same superposition method is performed in the $\left(x^{\prime}, y^{\prime}\right)$-coordinate system. The incident stress field can be described in the $\left(x^{\prime}, y^{\prime}\right)$-coordinate system as

$$
\bar{\tau}_{Y^{\prime} Z^{\prime}}^{\mathrm{i}}\left(X^{\prime}, Y^{\prime}, s\right)=\frac{\operatorname{sgn}\left(Y^{\prime}-H_{2}^{\prime}\right)}{2 \pi \mathrm{i}} \int_{\Gamma_{\lambda}} P \exp \left\{-s \alpha(\lambda)\left|Y^{\prime}-H_{2}^{\prime}\right|+s \lambda\left(X^{\prime}-H_{1}^{\prime}\right)\right\} \mathrm{d} \lambda,
$$

where

$$
H_{1}^{\prime}=-l-h_{1}+\frac{C_{45}}{C_{44}} h_{2}, \quad H_{2}^{\prime}=\frac{C_{e}}{C_{44}} h_{2} .
$$

By setting $Y^{\prime}=0$ and using the fundamental solutions presented in $\S 2$, the diffracted field for the $B$ wave (the first wave diffracted from crack tip B) can be constructed as follows

$$
\begin{aligned}
\bar{W}^{\prime \mathrm{B}}\left(X^{\prime}, Y^{\prime}, s\right)= & \frac{1}{2 \pi \mathrm{i}} \int_{\Gamma_{\eta_{1}}} P \exp \left\{-s\left[\alpha\left(\eta_{1}\right) H_{2}^{\prime}+\eta_{1} H_{1}^{\prime}\right]\right\} \\
& \times\left\{\frac{1}{2 \pi \mathrm{i}} \int_{\Gamma_{\eta_{2}}} \frac{-\operatorname{sgn}\left(Y^{\prime}\right) \mathrm{e}^{-s\left[\alpha\left(\eta_{2}\right)\left|Y^{\prime}\right|-\eta_{2} X^{\prime}\right]}}{C_{e} s \alpha_{+}\left(\eta_{1}\right)\left(\eta_{1}-\eta_{2}\right) \alpha_{-}\left(\eta_{2}\right)} \mathrm{d} \eta_{2}\right\} \mathrm{d} \eta_{1} \\
= & \frac{-\operatorname{sgn}\left(Y^{\prime}\right)}{(2 \pi \mathrm{i})^{2}} \int_{\Gamma_{\eta_{1}}} \int_{\Gamma_{\eta_{2}}} \frac{P \exp \left\{-s\left[\alpha\left(\eta_{1}\right) H_{2}^{\prime}+\eta_{1} H_{1}^{\prime}\right]\right\}}{C_{e} s \alpha_{+}\left(\eta_{1}\right)\left(\eta_{1}-\eta_{2}\right) \alpha_{-}\left(\eta_{2}\right)} \mathrm{d} \eta_{2} \mathrm{~d} \eta_{1},
\end{aligned}
$$

Proc. R. Soc. A (2005) 


$$
\begin{aligned}
\bar{\tau}_{Y^{\prime} Z^{\prime}}^{\mathrm{B}}\left(X^{\prime}, Y^{\prime}, s\right) & =\frac{1}{(2 \pi \mathrm{i})^{2}} \int_{\Gamma_{\eta_{1}}} \int_{\Gamma_{\eta_{2}}} \frac{P \alpha_{+}\left(\eta_{2}\right) \exp \left\{-s\left[\alpha\left(\eta_{1}\right) H_{2}^{\prime}+\eta_{1} H_{1}^{\prime}\right]\right\}}{\times \exp \left\{-s\left[\alpha\left(\eta_{2}\right)\left|Y^{\prime}\right|-\eta_{2} X^{\prime}\right]\right\}} \mathrm{d} \eta_{2} \mathrm{~d} \eta_{1}, \\
\alpha_{+}\left(\eta_{1}\right)\left(\eta_{1}-\eta_{2}\right) & \\
\bar{\tau}_{X^{\prime} Z^{\prime}}^{\mathrm{B}}\left(X^{\prime}, Y^{\prime}, s\right) & =\frac{-\operatorname{sgn}\left(Y^{\prime}\right)}{(2 \pi \mathrm{i})^{2}} \int_{\Gamma_{\eta_{1}}} \int_{\Gamma_{\eta_{2}}} \frac{\begin{array}{c}
P \eta_{2} \exp \left\{-s\left[\alpha\left(\eta_{1}\right) H_{2}^{\prime}+\eta_{1} H_{1}^{\prime}\right]\right\} \\
\times \exp \left\{-s\left[\alpha\left(\eta_{2}\right)\left|Y^{\prime}\right|-\eta_{2} X^{\prime}\right]\right\}
\end{array}}{\alpha_{+}\left(\eta_{1}\right)\left(\eta_{1}-\eta_{2}\right) \alpha_{-}\left(\eta_{2}\right)} \mathrm{d} \eta_{2} \mathrm{~d} \eta_{1} .
\end{aligned}
$$

The Cagniard-de Hoop method of Laplace inversion is used, and the solutions in time domain are

$$
\begin{aligned}
& W^{\prime \mathrm{B}}\left(X^{\prime}, Y^{\prime}, t\right) \\
& =W^{\prime \mathrm{B}_{1}}\left(X^{\prime}, Y^{\prime}, t\right) \\
& =\frac{\operatorname{sgn}\left(Y^{\prime}\right) P}{2 \pi^{2} C_{e}} \int_{b R^{\prime}+b R_{\mathrm{B}}}^{t} \int_{b R_{\mathrm{B}}}^{\tau-b R^{\prime}} \operatorname{Re}\left[\frac{\left(\partial \eta_{1}^{+} / \partial t_{1}\right)\left(\partial \eta_{2}^{+} / \partial t_{2}\right)}{\alpha_{+}\left(\eta_{1}^{+}\right)\left(\eta_{1}^{+}-\eta_{2}^{+}\right) \alpha_{-}\left(\eta_{2}^{+}\right)}\right. \\
& \left.-\frac{\left(\partial \eta_{1}^{-} / \partial t_{1}\right)\left(\partial \eta_{2}^{+} / \partial t_{2}\right)}{\alpha_{+}\left(\eta_{1}^{-}\right)\left(\eta_{1}^{-}-\eta_{2}^{+}\right) \alpha_{-}\left(\eta_{2}^{+}\right)}\right]_{t=\tau} \mathrm{d} t_{1} \mathrm{~d} \tau \\
& \tau_{Y^{\prime} Z^{\prime}}^{\mathrm{B}}\left(X^{\prime}, Y^{\prime}, t\right) \\
& =\tau_{Y^{\prime} Z^{\prime}}^{\mathrm{B}_{1}}\left(X^{\prime}, Y^{\prime}, t\right) \\
& =\frac{-P}{2 \pi^{2}} \int_{b R_{\mathrm{B}}}^{t-b R^{\prime}} \operatorname{Re}\left[\frac{\alpha_{+}\left(\eta_{2}^{+}\right)\left(\partial \eta_{1}^{+} / \partial t_{1}\right)\left(\partial \eta_{2}^{+} / \partial t_{2}\right)}{\alpha_{+}\left(\eta_{1}^{+}\right)\left(\eta_{1}^{+}-\eta_{2}^{+}\right)}\right. \\
& \left.-\frac{\alpha_{+}\left(\eta_{2}^{+}\right)\left(\partial \eta_{1}^{-} / \partial t_{1}\right)\left(\partial \eta_{2}^{+} / \partial t_{2}\right)}{\alpha_{+}\left(\eta_{1}^{-}\right)\left(\eta_{1}^{-}-\eta_{2}^{+}\right)}\right] \mathrm{d} t_{1}, \\
& \tau_{X^{\prime} Z^{\prime}}^{\mathrm{B}}\left(X^{\prime}, Y^{\prime}, t\right) \\
& =\tau_{X^{\prime} Z^{\prime}}^{\mathrm{B}_{1}}\left(X^{\prime}, Y^{\prime}, t\right) \\
& =\frac{\operatorname{sgn}\left(Y^{\prime}\right) P}{2 \pi^{2}} \int_{b R_{\mathrm{B}}}^{t-b R^{\prime}} \operatorname{Re}\left[\eta_{2}^{+} \frac{\left(\partial \eta_{1}^{+} / \partial t_{1}\right)\left(\partial \eta_{2}^{+} / \partial t_{2}\right)}{\alpha_{+}\left(\eta_{1}^{+}\right)\left(\eta_{1}^{+}-\eta_{2}^{+}\right) \alpha_{-}\left(\eta_{2}^{+}\right)}\right. \\
& \left.-\frac{\eta_{2}^{+}\left(\partial \eta_{1}^{-} / \partial t_{1}\right)\left(\partial \eta_{2}^{+} / \partial t_{2}\right)}{\alpha_{+}\left(\eta_{1}^{-}\right)\left(\eta_{1}^{-}-\eta_{2}^{+}\right) \alpha_{-}\left(\eta_{2}^{+}\right)}\right] \mathrm{d} t_{1},
\end{aligned}
$$

where

$$
\begin{gathered}
\eta_{1}^{ \pm}=\frac{t_{1} \cos \Phi_{\mathrm{B}}}{R_{\mathrm{B}}} \pm \mathrm{i} \frac{\sin \Phi_{\mathrm{B}}}{R_{\mathrm{B}}}\left(t_{1}^{2}-b^{2} R_{\mathrm{B}}^{2}\right)^{1 / 2}, \quad \eta_{2}^{ \pm}=\frac{-t_{2} \cos \Phi^{\prime}}{R^{\prime}} \pm \mathrm{i} \frac{\sin \Phi^{\prime}}{R^{\prime}}\left(t_{2}^{2}-b^{2} R^{\prime 2}\right)^{1 / 2}, \\
R_{\mathrm{B}}=\left(H_{1}^{\prime 2}+H_{2}^{\prime 2}\right)^{1 / 2}, \quad \Phi_{\mathrm{B}}=\arccos \left(\frac{H_{1}^{\prime}}{R_{\mathrm{B}}}\right), \quad R^{\prime}=\left(X^{\prime 2}+Y^{\prime 2}\right)^{1 / 2}, \\
\Phi^{\prime}=\operatorname{sgn}\left(Y^{\prime}\right) \arccos \left(\frac{X^{\prime}}{R^{\prime}}\right), \quad t_{1}+t_{2}=t .
\end{gathered}
$$

Proc. R. Soc. A (2005) 
When the first diffracted $B$ wave arrives at the right tip A of the finite crack after some later time, it carries with it a discontinuous displacement in the $Z$-direction which violates the boundary condition for $X>0$. In order to satisfy the boundary condition that the displacement must be continuous for $X>0$, a distributed displacement is required to close the crack-opening displacement. The diffracted $B A$ wave will be induced when the $B$ wave arrives at crack tip A. If we change the representation of $B$ wave from the $\left(x^{\prime}, y^{\prime}\right)$ - to the $(X, Y)$-coordinate system, then the displacement which must be eliminated ahead of the right tip $\mathrm{A}$ is

$$
\begin{aligned}
& \bar{W}^{\mathrm{B}}(X, 0, s) \\
& \quad=\frac{\operatorname{sgn}(Y)}{(2 \pi \mathrm{i})^{2}} \int_{\Gamma_{\eta_{1}}} \int_{\Gamma_{\eta_{2}}} \frac{P \exp \left\{-s\left[\alpha\left(\eta_{1}\right) H_{2}^{\prime}+\eta_{1} H_{1}^{\prime}\right]\right\} \exp \left\{s \eta_{2}(X+l)\right\}}{C_{e} s \alpha_{+}\left(\eta_{1}\right)\left(\eta_{1}+\eta_{2}\right) \alpha_{-}\left(-\eta_{2}\right)} \mathrm{d} \eta_{2} \mathrm{~d} \eta_{1} .
\end{aligned}
$$

It is envisaged that the crack is semi-infinite and lies along the line $Y=0,-\infty<$ $X<0$. The diffracted $B A$ wave generated from crack tip A can then be obtained by superimposing the distributed displacement that is equal and opposite to (3.23) ahead of the tip $X>0$ in the Laplace transform domain, as follows:

$$
\begin{aligned}
\bar{W}^{\mathrm{BA}}(X, Y, s) & \frac{-1}{(2 \pi \mathrm{i})^{2}} \int_{\Gamma_{\eta_{1}}} \int_{\Gamma_{\eta_{2}}} \frac{P \exp \left\{-s\left[\alpha\left(\eta_{1}\right) H_{2}^{\prime}+\eta_{1} H_{1}^{\prime}\right]\right\} \exp \left\{s \eta_{2} l\right\}}{C_{e} s \alpha_{+}\left(\eta_{1}\right)\left(\eta_{1}+\eta_{2}\right) \alpha_{-}\left(-\eta_{2}\right)} \\
& \times\left\{\frac{1}{2 \pi \mathrm{i}} \int_{\Gamma_{\eta_{3}}} \frac{-\operatorname{sgn}(Y) \alpha_{-}\left(\eta_{2}\right) \exp \left\{-s\left[\alpha\left(\eta_{3}\right)|Y|-\eta_{3} X\right]\right\}}{\left(\eta_{2}-\eta_{3}\right) \alpha_{-}\left(\eta_{3}\right)} \mathrm{d} \eta_{3}\right\} \mathrm{d} \eta_{2} \mathrm{~d} \eta_{1} \\
= & \frac{\operatorname{sgn}(Y)}{(2 \pi \mathrm{i})^{3}} \int_{\Gamma_{\eta_{1}}} \int_{\Gamma_{\eta_{2}}} \int_{\Gamma_{\eta_{3}}} \frac{P \alpha_{-}\left(\eta_{2}\right) \exp \left\{-s\left[\alpha\left(\eta_{1}\right) H_{2}^{\prime}+\eta_{1} H_{1}^{\prime}\right]\right\}}{C_{e} s \alpha_{+}\left(\eta_{1}\right)\left(\eta_{1}+\eta_{2}\right) \alpha_{-}\left(-\eta_{2}\right)\left(\eta_{2}-\eta_{3}\right) \alpha_{-}\left(\eta_{3}\right)} \mathrm{d} \eta_{3} \mathrm{~d} \eta_{2} \mathrm{~d} \eta_{1},
\end{aligned}
$$

$\bar{\tau}_{Y Z}^{\mathrm{B}} A(X, Y, s)$

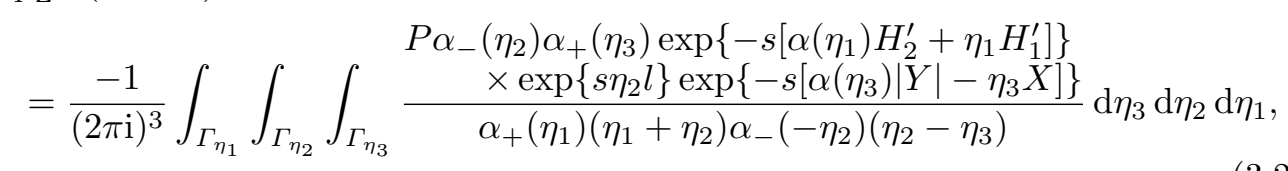

$$
\begin{aligned}
& \bar{\tau}_{X Z}^{\mathrm{BA}}(X, Y, s) \\
& \quad=\frac{\operatorname{sgn}(Y)}{(2 \pi \mathrm{i})^{3}} \int_{\Gamma_{\eta_{1}}} \int_{\Gamma_{\eta_{2}}} \int_{\Gamma_{\eta_{3}}} \frac{P \alpha_{-}\left(\eta_{2}\right) \eta_{3} \exp \left\{-s\left[\alpha\left(\eta_{1}\right) H_{2}^{\prime}+\eta_{1} H_{1}^{\prime}\right]\right\}}{\alpha_{+}\left(\eta_{1}\right)\left(\eta_{1}+\eta_{2}\right) \alpha_{-}\left(-\eta_{2}\right)\left(\eta_{2}-\eta_{3}\right) \alpha_{-}\left(\eta_{3}\right)} \mathrm{d} \eta_{3} \mathrm{~d} \eta_{2} \mathrm{~d} \eta_{1} .
\end{aligned}
$$

Here the fundamental solutions (2.28)-(2.30) are used for constructing the diffracted $B A$ wave (the second diffracted wave induced by the tip A). Next, inverting the 
Laplace transform of (3.24)-(3.26) yields

$$
\begin{aligned}
& W^{\mathrm{BA}}(X, Y, t) \\
& =W^{\mathrm{A}_{2}}(X, Y, t) \\
& =\frac{-\operatorname{sgn}(Y) P}{2 \pi^{3} C_{e}} \int_{b R+b R_{\mathrm{B}}+b l}^{t} \int_{b R_{\mathrm{B}}}^{\tau-b R-b l} \\
& \quad \int_{b l}^{\tau-b R-t_{1}} \operatorname{Im}\left[\frac{G\left(\eta_{1}^{+}, \eta_{2}^{+}\right)\left(\partial \eta_{1}^{+} / \partial t_{1}\right)\left(\partial \eta_{2}^{+} / \partial t_{2}\right)\left(\partial \eta_{3}^{+} / \partial t_{3}\right)}{\alpha_{+}\left(\eta_{1}^{+}\right)\left(\eta_{2}^{+}-\eta_{3}^{+}\right) \alpha_{-}\left(\eta_{3}^{+}\right)}\right. \\
& \left.\quad-\frac{G\left(\eta_{1}^{-}, \eta_{2}^{+}\right)\left(\partial \eta_{1}^{-} / \partial t_{1}\right)\left(\partial \eta_{2}^{+} / \partial t_{2}\right)\left(\partial \eta_{3}^{+} / \partial t_{3}\right)}{\alpha_{+}\left(\eta_{1}^{-}\right)\left(\eta_{2}^{+}-\eta_{3}^{+}\right) \alpha_{-}\left(\eta_{3}^{+}\right)}\right]_{t=\tau} \mathrm{d} t_{2} \mathrm{~d} t_{1} \mathrm{~d} \tau,
\end{aligned}
$$

$$
\begin{aligned}
& \tau_{Y Z}^{\mathrm{BA}}(X, Y, t) \\
& =\tau_{Y Z}^{\mathrm{A}_{2}}(X, Y, t) \\
& =\frac{P}{2 \pi^{3}} \int_{b R_{\mathrm{B}}}^{\tau-b R-b l} \\
& \quad \int_{b l}^{t-b R-t_{1}} \operatorname{Im}\left[\frac{G\left(\eta_{1}^{+}, \eta_{2}^{+}\right) \alpha_{+}\left(\eta_{3}^{+}\right)\left(\partial \eta_{1}^{+} / \partial t_{1}\right)\left(\partial \eta_{2}^{+} / \partial t_{2}\right)\left(\partial \eta_{3}^{+} / \partial t_{3}\right)}{\alpha_{+}\left(\eta_{1}^{+}\right)\left(\eta_{2}^{+}-\eta_{3}^{+}\right)}\right. \\
& \left.\quad-\frac{G\left(\eta_{1}^{-}, \eta_{2}^{+}\right) \alpha_{+}\left(\eta_{3}^{+}\right)\left(\partial \eta_{1}^{-} / \partial t_{1}\right)\left(\partial \eta_{2}^{+} / \partial t_{2}\right)\left(\partial \eta_{3}^{+} / \partial t_{3}\right)}{\alpha_{+}\left(\eta_{1}^{-}\right)\left(\eta_{2}^{+}-\eta_{3}^{+}\right)}\right] \mathrm{d} t_{2} \mathrm{~d} t_{1},
\end{aligned}
$$

$$
\begin{aligned}
& \tau_{X Z}^{\mathrm{BA}}(X, Y, t) \\
& =\tau_{X Z}^{\mathrm{A}_{2}}(X, Y, t) \\
& =\frac{-\operatorname{sgn}(Y) P}{2 \pi^{3}} \int_{b R_{\mathrm{B}}}^{\tau-b R-b l} \\
& \quad \int_{b l}^{t-b R-t_{1}} \operatorname{Im}\left[\frac{G\left(\eta_{1}^{+}, \eta_{2}^{+}\right) \eta_{3}^{+}\left(\partial \eta_{1}^{+} / \partial t_{1}\right)\left(\partial \eta_{2}^{+} / \partial t_{2}\right)\left(\partial \eta_{3}^{+} / \partial t_{3}\right)}{\alpha_{+}\left(\eta_{1}^{+}\right)\left(\eta_{2}^{+}-\eta_{3}^{+}\right) \alpha_{-}\left(\eta_{3}^{+}\right)}\right. \\
& \left.\quad-\frac{G\left(\eta_{1}^{-}, \eta_{2}^{+}\right) \eta_{3}^{+}\left(\partial \eta_{1}^{-} / \partial t_{1}\right)\left(\partial \eta_{2}^{+} / \partial t_{2}\right)\left(\partial \eta_{3}^{+} / \partial t_{3}\right)}{\alpha_{+}\left(\eta_{1}^{-}\right)\left(\eta_{2}^{+}-\eta_{3}^{+}\right) \alpha_{-}\left(\eta_{3}^{+}\right)}\right] \mathrm{d} t_{2} \mathrm{~d} t_{1},
\end{aligned}
$$

where

$$
\begin{gathered}
G\left(\eta_{1}, \eta_{2}\right)=\frac{\alpha_{-}\left(\eta_{2}\right)}{\left(\eta_{1}+\eta_{2}\right) \alpha_{-}\left(-\eta_{2}\right)}, \quad \eta_{1}^{ \pm}=\frac{t_{1} \cos \Phi_{\mathrm{B}}}{R_{\mathrm{B}}} \pm \mathrm{i} \frac{\sin \Phi_{\mathrm{B}}}{R_{\mathrm{B}}}\left(t_{1}^{2}-b^{2} R_{\mathrm{B}}^{2}\right)^{1 / 2}, \\
\eta_{2}^{ \pm}=\frac{-t_{2}}{l} \pm \mathrm{i} \varepsilon, \quad \eta_{3}^{ \pm}=\frac{-t_{3} \cos \Phi}{R} \pm \mathrm{i} \frac{\sin \Phi}{R}\left(t_{3}^{2}-b^{2} R^{2}\right)^{1 / 2}, \\
R_{\mathrm{B}}=\left(H_{1}^{\prime 2}+H_{2}^{\prime 2}\right)^{1 / 2}, \quad \Phi_{\mathrm{B}}=\arccos \left(\frac{H_{1}^{\prime}}{R_{\mathrm{B}}}\right), \quad R=\left(X^{2}+Y^{2}\right)^{1 / 2},
\end{gathered}
$$

Proc. R. Soc. A (2005) 


$$
\Phi=\operatorname{sgn}(Y) \arccos (X / R), \quad t_{1}+t_{2}+t_{3}=t .
$$

Similarly, the diffracted AB wave (the second diffracted wave induced by the tip B) can be obtained from (3.10) and (2.28)-(2.30) if the same procedure is used, and the final results are

$$
\begin{aligned}
& W^{\prime \mathrm{AB}}\left(X^{\prime}, Y^{\prime}, t\right) \\
& =W^{\prime \mathrm{B}_{2}}\left(X^{\prime}, Y^{\prime}, t\right) \\
& =\frac{\operatorname{sgn}\left(Y^{\prime}\right) P}{2 \pi^{3} C_{e}} \int_{b R^{\prime}+b R_{\mathrm{A}}+b l}^{t} \int_{b R_{\mathrm{A}}}^{\tau-b R^{\prime}-b l} \\
& \int_{b l}^{\tau-b R^{\prime}-t_{1}} \operatorname{Im}\left[\frac{G\left(\eta_{1}^{+}, \eta_{2}^{+}\right)\left(\partial \eta_{1}^{+} / \partial t_{1}\right)\left(\partial \eta_{2}^{+} / \partial t_{2}\right)\left(\partial \eta_{3}^{+} / \partial t_{3}\right)}{\alpha_{+}\left(\eta_{1}^{+}\right)\left(\eta_{2}^{+}-\eta_{3}^{+}\right) \alpha_{-}\left(\eta_{3}^{+}\right)}\right. \\
& \left.-\frac{G\left(\eta_{1}^{-}, \eta_{2}^{+}\right)\left(\partial \eta_{1}^{-} / \partial t_{1}\right)\left(\partial \eta_{2}^{+} / \partial t_{2}\right)\left(\partial \eta_{3}^{+} / \partial t_{3}\right)}{\alpha_{+}\left(\eta_{1}^{-}\right)\left(\eta_{2}^{+}-\eta_{3}^{+}\right) \alpha_{-}\left(\eta_{3}^{+}\right)}\right]_{t=\tau} \mathrm{d} t_{2} \mathrm{~d} t_{1} \mathrm{~d} \tau, \\
& \tau_{Y^{\prime} Z^{\prime}}^{\mathrm{AB}}\left(X^{\prime}, Y^{\prime}, t\right) \\
& =\tau_{Y^{\prime} Z^{\prime}}^{\mathrm{B}_{2}}\left(X^{\prime}, Y^{\prime}, t\right) \\
& =\frac{-P}{2 \pi^{3}} \int_{b R_{\mathrm{A}}}^{\tau-b R^{\prime}-b l} \\
& \int_{b l}^{t-b R^{\prime}-t_{1}} \operatorname{Im}\left[\frac{G\left(\eta_{1}^{+}, \eta_{2}^{+}\right) \alpha_{+}\left(\eta_{3}^{+}\right)\left(\partial \eta_{1}^{+} / \partial t_{1}\right)\left(\partial \eta_{2}^{+} / \partial t_{2}\right)\left(\partial \eta_{3}^{+} / \partial t_{3}\right)}{\alpha_{+}\left(\eta_{1}^{+}\right)\left(\eta_{2}^{+}-\eta_{3}^{+}\right)}\right. \\
& \left.-\frac{G\left(\eta_{1}^{-}, \eta_{2}^{+}\right) \alpha_{+}\left(\eta_{3}^{+}\right)\left(\partial \eta_{1}^{-} / \partial t_{1}\right)\left(\partial \eta_{2}^{+} / \partial t_{2}\right)\left(\partial \eta_{3}^{+} / \partial t_{3}\right)}{\alpha_{+}\left(\eta_{1}^{-}\right)\left(\eta_{2}^{+}-\eta_{3}^{+}\right)}\right] \mathrm{d} t_{2} \mathrm{~d} t_{1} \text {, } \\
& \tau_{X^{\prime} Z^{\prime}}^{\mathrm{AB}}\left(X^{\prime}, Y^{\prime}, t\right) \\
& =\tau_{X^{\prime} Z^{\prime}}^{\mathrm{B}_{2}}\left(X^{\prime}, Y^{\prime}, t\right) \\
& =\frac{\operatorname{sgn}(Y) P}{2 \pi^{3}} \int_{b R_{\mathrm{A}}}^{\tau-b R^{\prime}-b l} \\
& \int_{b l}^{t-b R^{\prime}-t_{1}} \operatorname{Im}\left[\frac{G\left(\eta_{1}^{+}, \eta_{2}^{+}\right) \eta_{3}^{+}\left(\partial \eta_{1}^{+} / \partial t_{1}\right)\left(\partial \eta_{2}^{+} / \partial t_{2}\right)\left(\partial \eta_{3}^{+} / \partial t_{3}\right)}{\alpha_{+}\left(\eta_{1}^{+}\right)\left(\eta_{2}^{+}-\eta_{3}^{+}\right) \alpha_{-}\left(\eta_{3}^{+}\right)}\right. \\
& \left.-\frac{G\left(\eta_{1}^{-}, \eta_{2}^{+}\right) \eta_{3}^{+}\left(\partial \eta_{1}^{-} / \partial t_{1}\right)\left(\partial \eta_{2}^{+} / \partial t_{2}\right)\left(\partial \eta_{3}^{+} / \partial t_{3}\right)}{\alpha_{+}\left(\eta_{1}^{-}\right)\left(\eta_{2}^{+}-\eta_{3}^{+}\right) \alpha_{-}\left(\eta_{3}^{+}\right)}\right] \mathrm{d} t_{2} \mathrm{~d} t_{1},
\end{aligned}
$$

where

$$
\begin{gathered}
\eta_{1}^{ \pm}=\frac{t_{1} \cos \Phi_{\mathrm{A}}}{R_{\mathrm{A}}} \pm \mathrm{i} \frac{\sin \Phi_{\mathrm{A}}}{R_{\mathrm{A}}}\left(t_{1}^{2}-b^{2} R_{\mathrm{A}}^{2}\right)^{1 / 2}, \quad \eta_{2}^{ \pm}=\frac{-t_{2}}{l} \pm \mathrm{i} \varepsilon, \\
\eta_{3}^{ \pm}=\frac{-t_{3} \cos \Phi^{\prime}}{R^{\prime}} \pm \mathrm{i} \frac{\sin \Phi^{\prime}}{R^{\prime}}\left(t_{3}^{2}-b^{2} R^{\prime 2}\right)^{1 / 2}, \quad t_{1}+t_{2}+t_{3}=t, \quad R_{\mathrm{A}}=\left(H_{1}^{2}+H_{2}^{2}\right)^{1 / 2},
\end{gathered}
$$

Proc. R. Soc. A (2005) 


$$
\Phi_{\mathrm{A}}=\arccos \left(\frac{H_{1}}{R_{\mathrm{A}}}\right), \quad R^{\prime}=\left(X^{\prime 2}+Y^{\prime 2}\right)^{1 / 2}, \quad \Phi^{\prime}=\operatorname{sgn}\left(Y^{\prime}\right) \arccos \left(\frac{X^{\prime}}{R^{\prime}}\right) .
$$

The AB wave, which is generated by the diffracted A wave from the left crack tip B, will return to the tip A at a later time. After it arrives at the tip A, the boundary condition for $X>0$ will not be satisfied. According to the above-mentioned process, an appropriate sequence of displacement along $X>0$ must be superimposed to close the opening displacement ahead of crack tip A. By using the fundamental solutions of the distributed displacement, the diffracted ABA wave (the third diffracted wave induced by the tip A) can be constructed. Similarly, when the diffracted BA wave reaches crack tip $\mathrm{B}$, the induced $\mathrm{BAB}$ wave (the third diffracted wave induced by the tip B) can be obtained in the same method. Following a similar procedure continuously, the complete full-field solutions that account for the contributions of all the diffracted waves induced from two crack tips are finally derived. The complete transient solutions for displacement and shear stresses can be expressed explicitly in compact formulations as

$$
\begin{gathered}
W(X, Y, t)=W^{\mathrm{i}}(X, Y, t)+W^{r}(X, Y, t)+\sum_{n=1}^{\infty}\left[W^{\mathrm{A}_{n}}(X, Y, t)+W^{\prime \mathrm{B}_{n}}\left(X^{\prime}, Y^{\prime}, t\right)\right] \\
\tau_{Y Z}(X, Y, t)=\tau_{Y Z}^{\mathrm{i}}(X, Y, t)+\tau_{Y Z}^{r}(X, Y, t)+\sum_{n=1}^{\infty}\left[\tau_{Y Z}^{\mathrm{A}_{n}}(X, Y, t)+\tau_{Y^{\prime} Z^{\prime}}^{\mathrm{B}_{n}}\left(X^{\prime}, Y^{\prime}, t\right)\right] \\
\tau_{X Z}(X, Y, t)=\tau_{X Z}^{\mathrm{i}}(X, Y, t)+\tau_{X Z}^{r}(X, Y, t)+\sum_{n=1}^{\infty}\left[\tau_{X Z}^{\mathrm{A}_{n}}(X, Y, t)+\tau_{X^{\prime} Z^{\prime}}^{\mathrm{B}_{n}}\left(X^{\prime}, Y^{\prime}, t\right)\right] .
\end{gathered}
$$

The details of functions presented in (3.33)-(3.35) for the contributions of incident, reflected and diffracted waves are given in Appendix A.

It should be noted that the solutions shown in (3.33)-(3.35) are expressed in the transformed $(X, Y)$ - and $\left(x^{\prime}, y^{\prime}\right)$-coordinate systems. The transient solutions for the anisotropic finite crack in the original coordinate system of $(x, y)$ can be obtained from (2.9), (2.13) and (2.14) in which the coordinate relations expressed in (2.4) and (2.5) are used. Though the number of terms in (3.33)-(3.35) is infinity, only a finite number of terms will be non-zero in the series solution during a finite observation time. Each term in the solution represents the contribution of a wave and the transient solution is exact up to the arrival time of the next wave.

\section{Transient solutions of dynamic stress-intensity factors}

The transient full-field solutions for shear stresses and out-of-plane displacement have been derived in the previous section. The dynamic stress-intensity factor of a finite crack in an anisotropic medium subjected to concentrated impact loadings can be obtained by using a similar methodology. In order to derive the solution of the dynamic stress-intensity factor in the Laplace transform domain, the fundamental solutions in (2.24) and (2.31) should be implemented. The inverse Laplace transform can also be performed by using the Cagniard-de Hoop method. The results of 


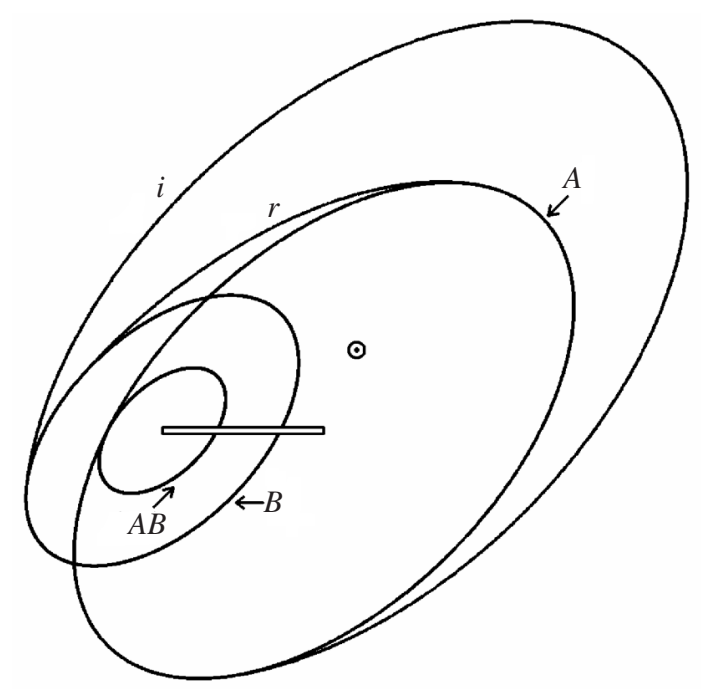

Figure 3. Wavefronts of the incident, reflected and diffracted waves for a short time period after applying a dynamic body force in an anisotropic medium.

dynamic stress-intensity factors in time domain at the right tip A can be expressed explicitly as

$$
K^{\mathrm{A}}(t)=\sum_{n=1}^{\infty} K^{\mathrm{A}_{n}}(t),
$$

where

$$
\begin{aligned}
K^{\mathrm{A}_{1}}(t)= & P \sqrt{\frac{2}{\pi R_{\mathrm{A}}}} \sin \left(\frac{1}{2} \Phi_{\mathrm{A}}\right) \mathcal{H}\left(t-b R_{\mathrm{A}}\right) \\
K^{\mathrm{A}_{n}}(t)= & \frac{\sqrt{2} P(i)^{q}}{2 \sqrt{\pi} \pi^{n}(i)^{n}} \int_{b R_{0}+(n-1) b l}^{t} \int_{b R_{0}}^{c_{1}} \int_{b l}^{c_{2}} \\
& \cdots \int_{b l}^{c_{n-1}} \frac{1}{\sqrt{t-\tau}} \mathrm{OP}[\mathrm{SIF}]_{t=\tau} \mathrm{d} t_{n-1} \mathrm{~d} t_{n-2} \cdots \mathrm{d} t_{1} \mathrm{~d} \tau
\end{aligned}
$$

for $n=2,3,4, \ldots$.

Details of functions and parameters presented in (4.3) are given in Appendix B.

The result for the dynamic stress-intensity factor for the incident wave arrives at crack tip A is indicated in (4.2) and is the well-known result for a semi-infinite crack in an unbounded medium. It is noted that the dynamic stress-intensity factor jumps from zero to the corresponding static value after the incident wave generated from the loading point arrives at crack tip A.

\section{Numerical results of transient responses}

In the previous sections, the transient analytical full-field solutions of shear stresses and displacement, and the dynamic stress-intensity factor, have been derived and expressed in explicit forms. The induced wavefronts of incident, reflected and diffracted waves in a short time period are plotted in figure 3 . The labels 'A' and ' $\mathrm{B}$ ' 


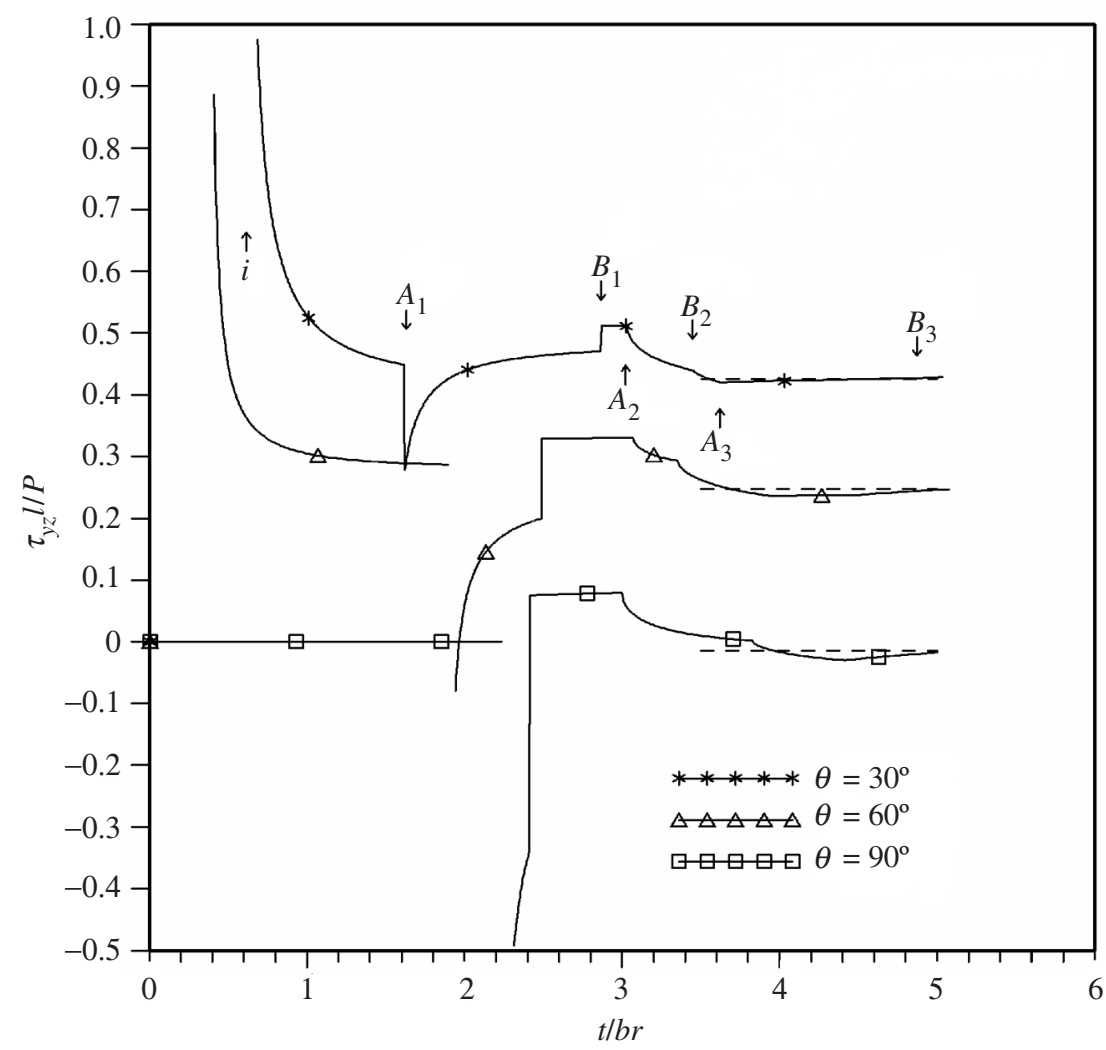

Figure 4. Transient shear stresses $\tau_{y z}$ for different values of $\theta$ due to impact loading applied at $(l, l) . C_{44}: C_{45}: C_{55}=1: 1: 2 ; h_{1} / l=1 ; h_{2} / l=1 ; r / l=1$.

in figure 3 are the first diffracted waves induced by crack tips A and B, respectively. When the diffracted A wave arrives at crack tip B, the diffracted 'AB' wave is induced and is also indicated in figure 3. Since the transient solution is exact up to the arrival time of the next wave, only a finite number of waves will be involved in the numerical calculation. However, the numerical calculation includes many high-dimensional integrals; the convergence of the integration should be carefully examined. These integrals have a square-root singularity at the arrival time of waves and only appear at one limit of integration. The singular behaviour near one limit of integration can be easily removed by the conventional scheme of changing integration variables. The program divides the interval of integration into $m$ segments and is calculated by the 24 -term Gaussian formula. The results show that the convergences of most cases can be achieved for $m \leqslant 16$. Numerical results are presented for the transient responses of shear stress $\tau_{y z}$ and dynamic stress-intensity factor $K^{\mathrm{A}}$ for crack tip A. The transient response of stress $\tau_{x z}$ is not presented because of its similar characteristics to $\tau_{y z}$. The corresponding static solutions of the anisotropic material with a finite crack are (Ting 1996)

$$
w^{s}(x, y)=\frac{-P}{\pi C_{e}} \operatorname{Re}\left[\ln \left(\varsigma-\varsigma_{0}\right)+\ln \left(\varsigma^{-1}-\bar{\varsigma}_{0}\right)\right],
$$




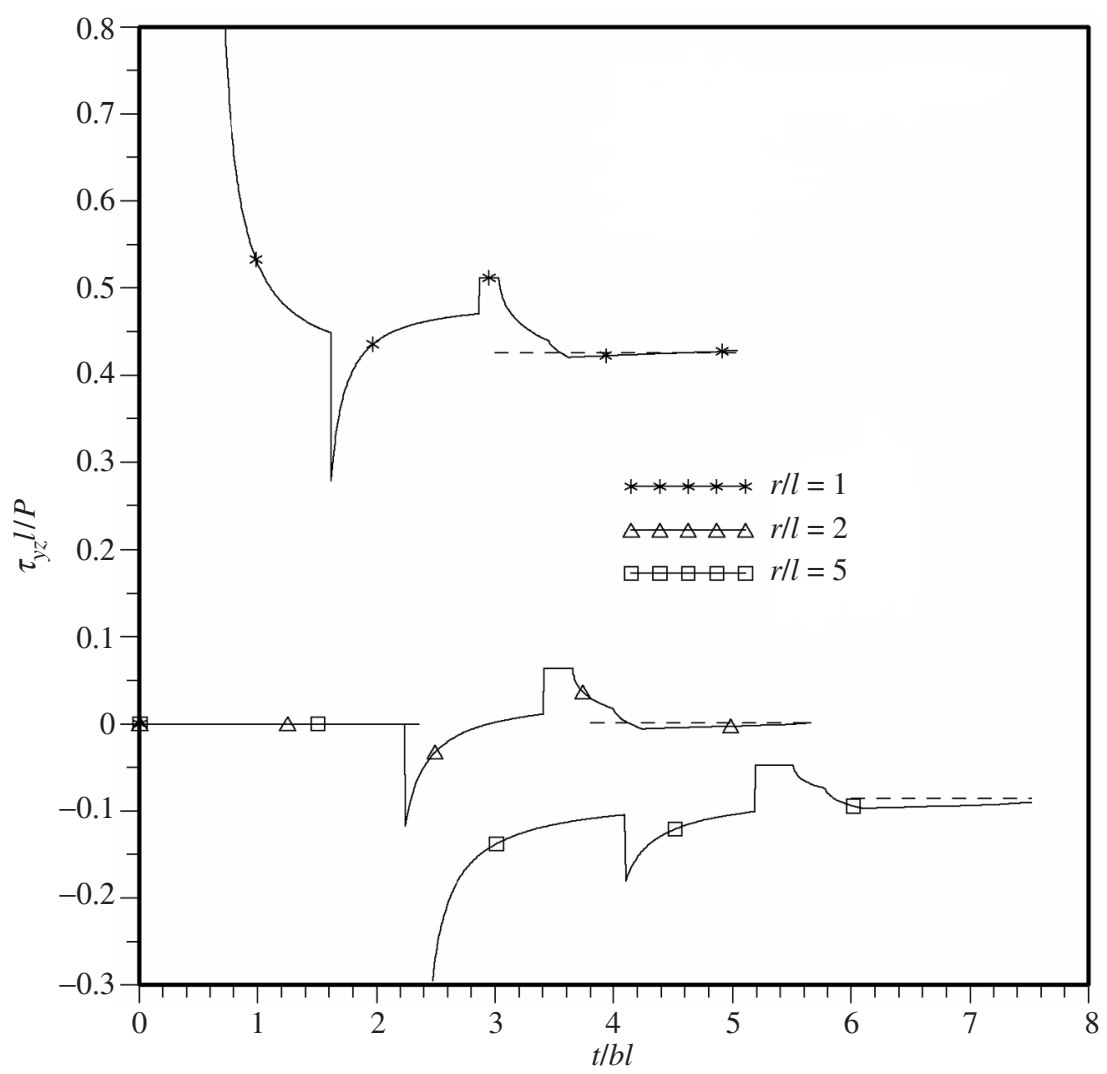

Figure 5. Transient shear stresses $\tau_{y z}$ for different values of $r$ due to impact loading applied at $(l, l) . C_{44}: C_{45}: C_{55}=1: 1: 2 ; h_{1} / l=1 ; h_{2} / l=1 ; r / l=1 ; \theta=30^{\circ}$.

$$
\begin{aligned}
\tau_{y z}^{s}(x, y) & =\frac{P}{\pi} \operatorname{Im}\left[\frac{1}{\sqrt{z^{2}-(l / 2)^{2}}}\left(\frac{\varsigma}{\varsigma-\varsigma_{0}}-\frac{\varsigma^{-1}}{\varsigma^{-1}-\bar{\varsigma}_{0}}\right)\right], \\
\tau_{x z}^{s}(x, y) & =\frac{-P}{\pi} \operatorname{Im}\left[\frac{p}{\sqrt{z^{2}-(l / 2)^{2}}}\left(\frac{\varsigma}{\varsigma-\varsigma_{0}}-\frac{\varsigma^{-1}}{\varsigma^{-1}-\bar{\varsigma}_{0}}\right)\right], \\
K^{\mathrm{A}, s} & =\frac{\sqrt{2} P}{\sqrt{\pi l}} \operatorname{Im}\left[\frac{1}{1-\varsigma_{0}}-\frac{1}{1-\bar{\varsigma}_{0}}\right],
\end{aligned}
$$

where $\zeta_{0}$ and $\bar{\zeta}_{0}$ are complex conjugates and

$$
\begin{gathered}
\varsigma_{0}=\frac{2}{l}\left[z_{0}+\sqrt{z_{0}^{2}-(l / 2)^{2}}\right], \quad \varsigma=\frac{2}{l}\left[z+\sqrt{z^{2}-(l / 2)^{2}}\right], \\
z_{0}=h_{1}+\frac{1}{2} l+p h_{2}, \quad z=x+\frac{1}{2} l+p y, \quad p=\frac{-C_{45}+\mathrm{i} C_{e}}{C_{44}} .
\end{gathered}
$$

The elastic constants with the ratio $C_{44}: C_{45}: C_{55}=1: 1: 2$ and the dynamic concentrated loading with Heaviside-function time dependence located at $\left(h_{1}, h_{2}\right)=(l, l)$ are selected first for analysing the transient response for various field points. Figures 4 and 5 plot the transient responses of shear stress $\tau_{y z}$ for different values of $\theta$ and $r$, respectively. The corresponding non-dimensional time of the incident and diffracted 


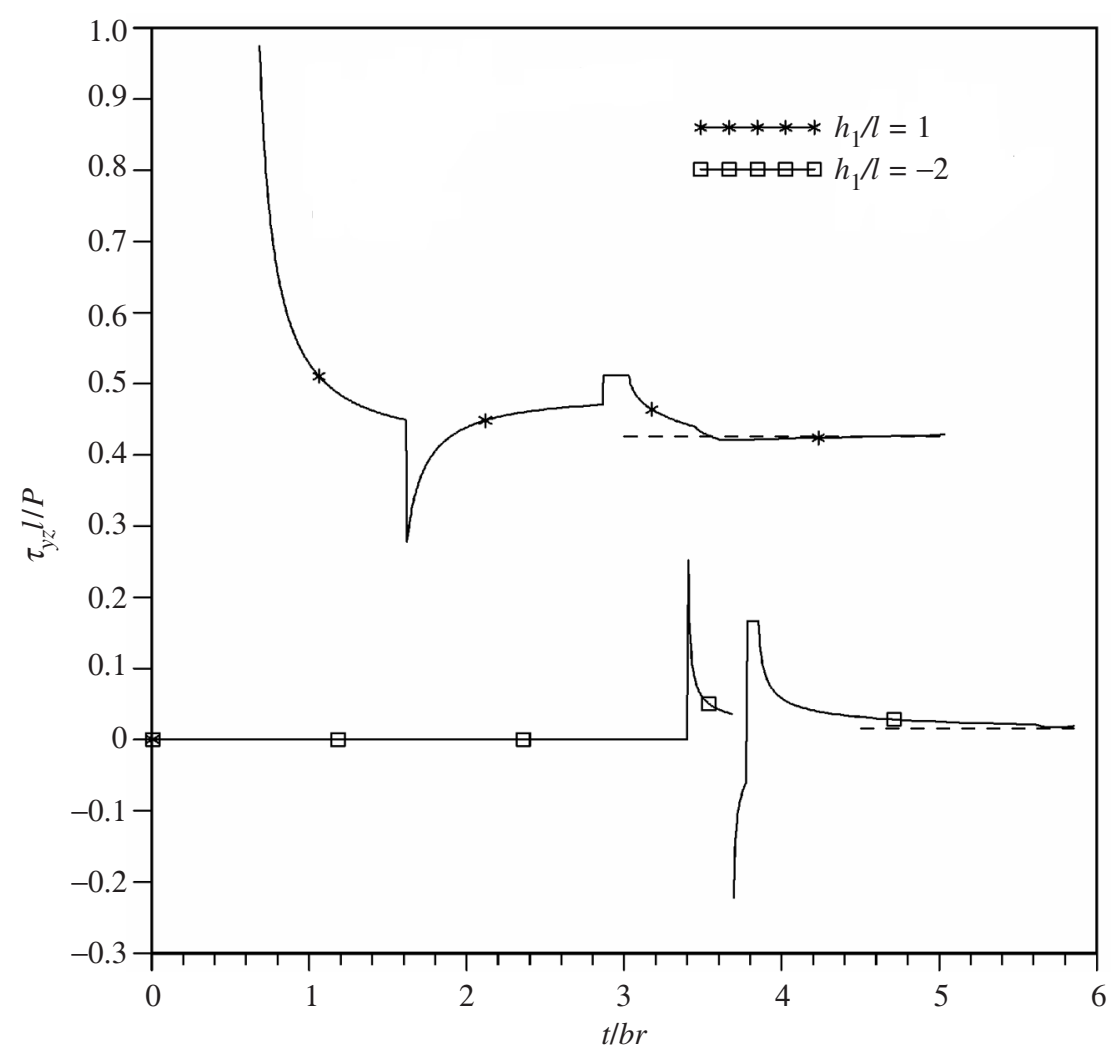

Figure 6. Transient shear stresses $\tau_{y z}$ for applying impact loading at $h_{1} / l=1$ and $-2 . C_{44}: C_{45}: C_{55}=1: 1: 2 ; h_{1} / l=1 ; h_{2} / l=1 ; r / l=1 ; \theta=30^{\circ}$.

waves which arrive at the field point for $\theta=30^{\circ}$ is indicated in figure 4 . The static solutions are also indicated in these figures by dashed lines. It is clearly shown in these two figures that the incident and reflected waves carry a square-root singularity at their wavefronts, while the diffracted waves ( $A_{1}$ and $B_{1}$ waves) have only finite jumps at their wavefronts. The singular behaviour of stress at wavefronts for the incident and reflected waves is clearly indicated in (3.6) and (3.14), respectively. It is worth noting that the transient stress tends toward the corresponding static value after the first eight waves (i.e. the $i, r, A_{1}, B_{1}, A_{2}, B_{2}, A_{3}$ and $B_{3}$ waves) have passed the specified field point. Figure 6 shows the transient response of shear stress for applied loading at different locations $h_{1} / l=1$ and -2 . It is also indicated that the transient response approaches the corresponding static solution after the $A_{3}$ or $B_{3}$ diffracted wave has passed the field point. The transient stresses for different ratios of elastic constants are plotted in figure 7 , in which the ratio $C_{44}: C_{45}: C_{55}=1: 0: 1$ represents the isotropic case. It can be seen that the transient response approaches the static value after the eighth wave has passed the field point.

Figure 8 shows the transient response of normalized stress-intensity factors $K l^{1 / 2} / P$ versus the dimensionless time $t / b l$ for different combinations of material constants. It is noted in this figure that the dynamic stress-intensity factor will reach a constant value when the incident wave arrives at crack tip A, which is greater than the static value, will remain constant before the first diffracted wave generated 


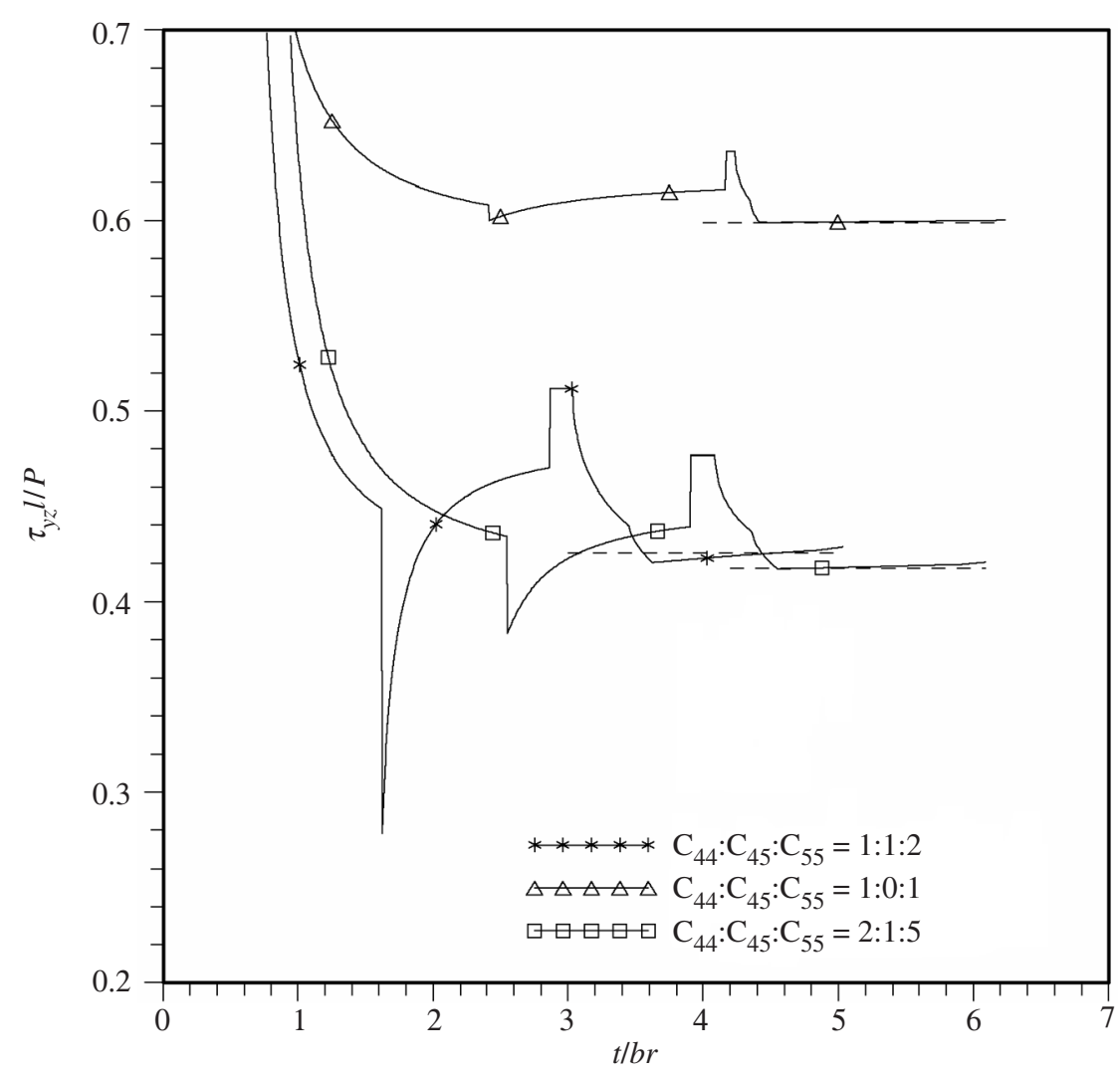

Figure 7. Transient shear stresses $\tau_{y z}$ for different ratios of elastic constants.

$$
h_{1} / l=1 ; h_{2} / l=1 ; r / l=1 ; \theta=30^{\circ} \text {. }
$$

by crack tip B arrives, and will then oscillate near the static value after the first three diffracted waves from crack tip B have passed through crack tip A. It is indicated in the previous section (equation (4.2)) that the constant value of the dynamic stress-intensity factor is the well-known result of the static solution for a semi-infinite crack. The isotropic case presented in figure 8 is chosen for a detailed explanation: the incident wave arrives at crack tip A at non-dimensional time $\sqrt{2}=1.41$ and the dynamic stress-intensity factor jumps from zero to the static solution of the semi-infinite crack with the value $(\sqrt{2} / \pi)^{1 / 2} \sin (\pi / 8)=0.257$ (i.e. from (4.2)). Then the diffracted $B_{1}$ wave generated from crack tip B arrives at crack tip A at the non-dimensional time $\sqrt{5}+1=3.24$ and the dynamic stress-intensity factor starts to decrease significantly to the correspondent static value of the finite crack. The diffracted $A_{1}$ wave travels along the crack face with two crack lengths (i.e. from crack tip A to crack tip B and back to crack tip A) and reaches crack tip A at the nondimensional time $\sqrt{2}+2=3.41$; the dynamic stress-intensity factor increases with a small magnitude until the next wave arrives at crack tip $\mathrm{A}$ at the non-dimensional time 5.24. Next, the impact loadings located at $h_{2} / l=1$ for various values of $h_{1} / l$ are used for calculation and the results are shown in figure 9 . In order to see the dynamic phenomenon more clearly, the non-dimensional stress-intensity factor presented in figure 9 is changed to $K^{\mathrm{A}} / K^{\mathrm{A} \text {,static }}$, and the result is plotted in figure 10 . It 


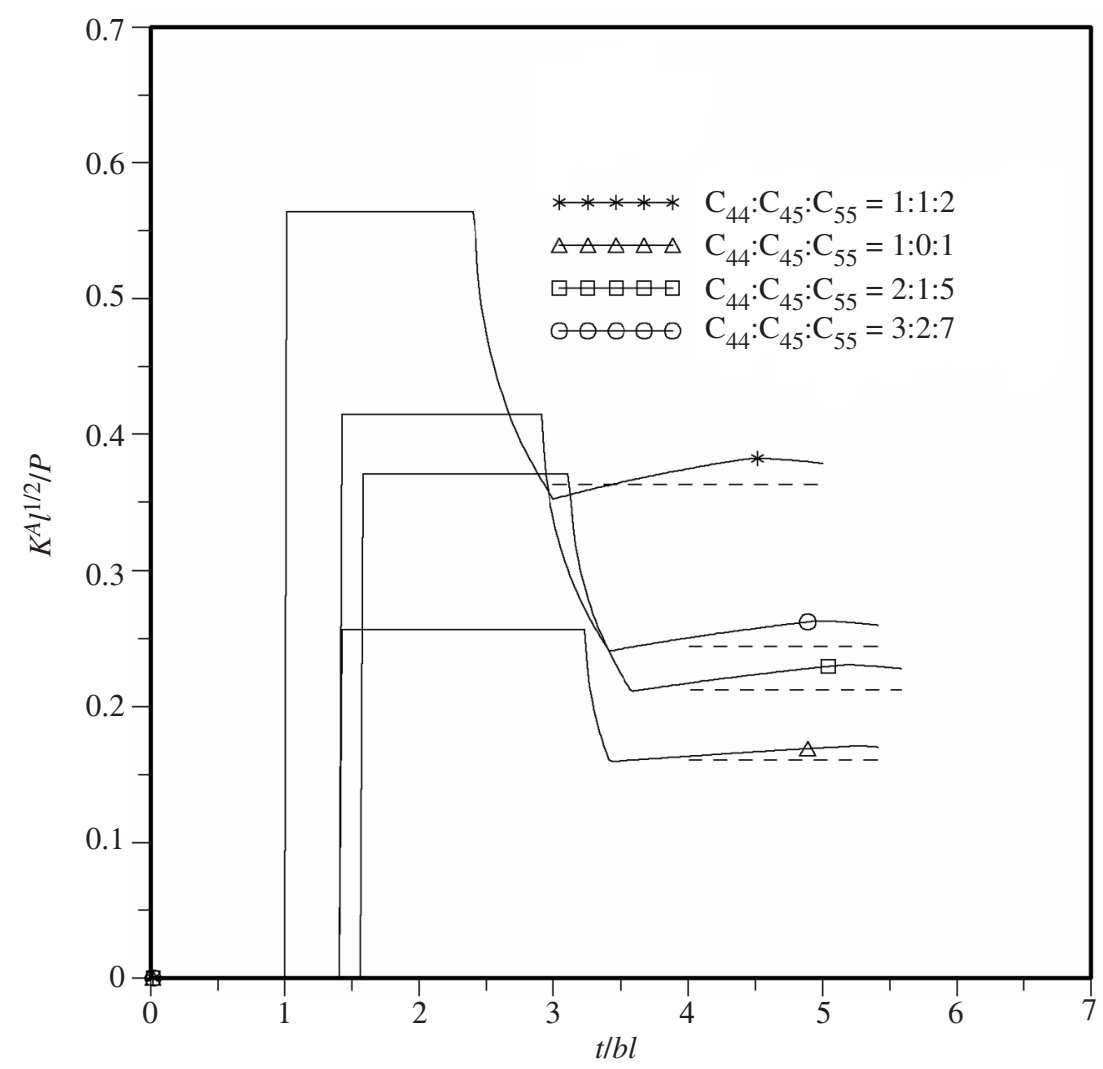

Figure 8. Transient responses of dimensionless stress-intensity factor $K^{\mathrm{A}} l^{1 / 2} / P$ for different ratios of elastic constants. $h_{1} / l=1 ; h_{2} / l=1$.

can be seen that the maximum dynamic overshoot always occurs at the arrival time of the incident wave at crack tip $\mathrm{A}$, and remains constant until the diffracted wave (i.e. $B_{1}$ wave) generated from the other crack tip arrives; it then starts to decrease and oscillate near the static value. Therefore, the ratio of the maximum dynamic value to the static value, which is usually defined as the elastodynamic overshoot of the stress-intensity factor, can be explicitly expressed as follows:

$$
\frac{K_{\max }^{\mathrm{A}}(t)}{K^{\mathrm{A}, \mathrm{static}}}=\frac{\sqrt{l} \sin \left(\frac{1}{2} \Phi_{\mathrm{A}}\right)}{\sqrt{R_{\mathrm{A}}} \operatorname{Im}\left[\left(1-\varsigma_{0}\right)^{-1}-\left(1-\bar{\varsigma}_{0}\right)^{-1}\right]} .
$$

Note that in the case of $h_{1} / l=-1$, as shown in figure 10, the value of dynamic overshoot is greater than five. Hence, the elastodynamic overshoot of the stressintensity factor becomes very significant. It is clearly indicated in figure 9 that the dynamic stress-intensity factor induced by the incident wave for different values of $h_{1} / l$ is not very different. After the $B_{1}$ wave generated from crack tip B has arrived crack tip A, the stress-intensity factor decreases near the correspondent static value. Since the loading location in the case of $h_{1} / l=-1$ has the shortest distance to crack tip B, the influence of $B_{1}$ wave on the stress-intensity factor of crack tip A is very significant and this is the reason why the dynamic overshoot is large for $h_{1} / l=-1$. Equation (5.5) provides a simple expression of the dynamic overshoot for 


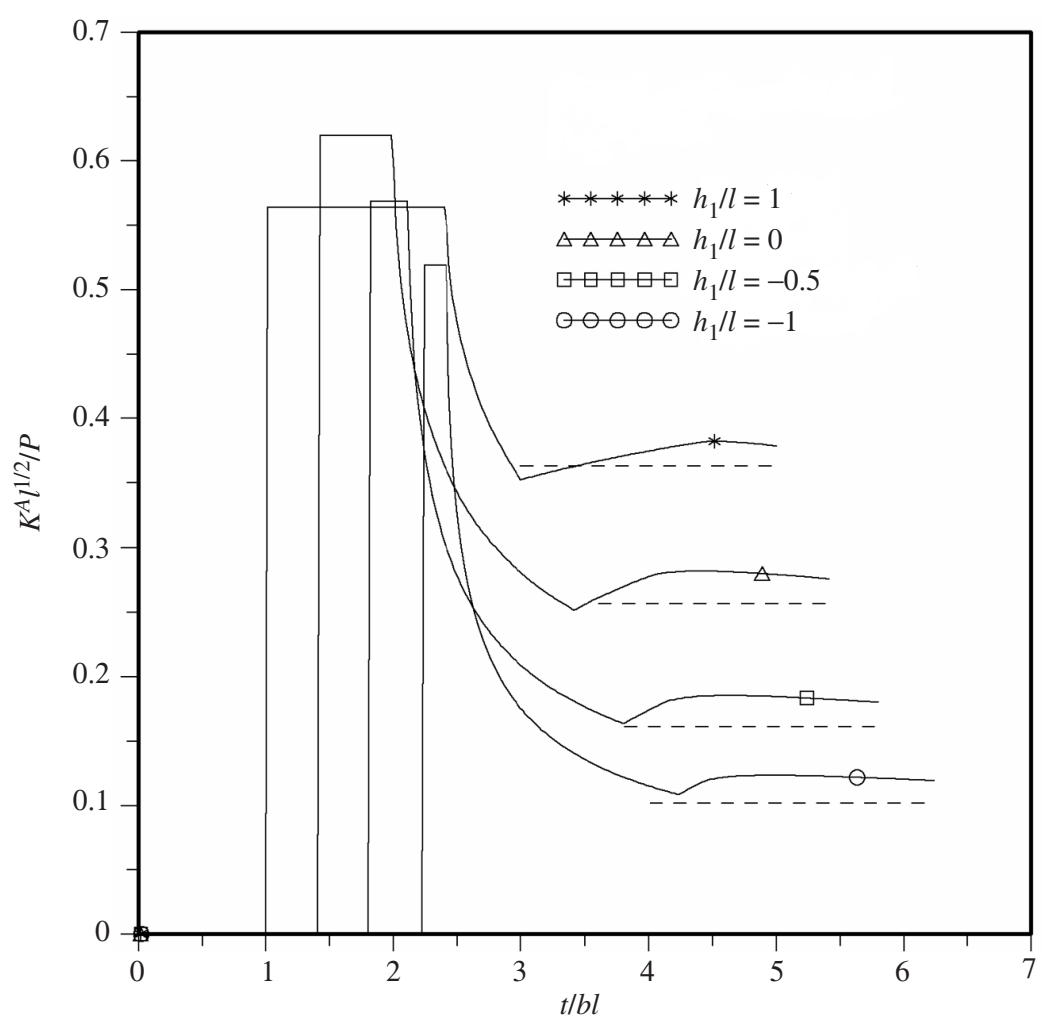

Figure 9. Transient responses of dimensionless stress-intensity factor $K^{\mathrm{A}} l^{1 / 2} / P$ for applying impact loading at $h_{2} / l=1$ for different values of $h_{1} / l$. $C_{44}: C_{45}: C_{55}=1: 1: 2$.

an anisotropic medium with a finite crack, which is dependent on the location of the applied loading, the crack length and material constants. This equation can be used for the safety consideration of crack in mechanical design.

\section{(a) Conclusions}

The transient problem of an anisotropic medium containing a finite crack subjected to dynamic anti-plane loading on the medium is investigated to gain insight into the phenomenon of the interaction of stress waves with cracks. A linear coordinate transformation is introduced in this study to simplify the problem. The linear coordinate transformation reduces the anisotropic problem to an equivalent isotropic problem with a similar geometry configuration. This problem contains characteristic lengths and is solved by superposition of two proposed fundamental solutions in the Laplace transform domain. The exact transient full-field solutions for shear stresses and displacement are explicitly expressed in closed forms. These solutions are valid for infinitely long time and have accounted for the contribution of incident, reflected and multiply diffracted waves. Every term in the solution has its own physical meaning. Transient solutions for dynamic stress-intensity factors are also obtained and expressed in explicit formulations. While the dynamic stress-intensity factor is of primary interest to dynamic fracture mechanics, the complete scattered stress and displacement fields are of particular interest to quantitative non-destructive evalu- 


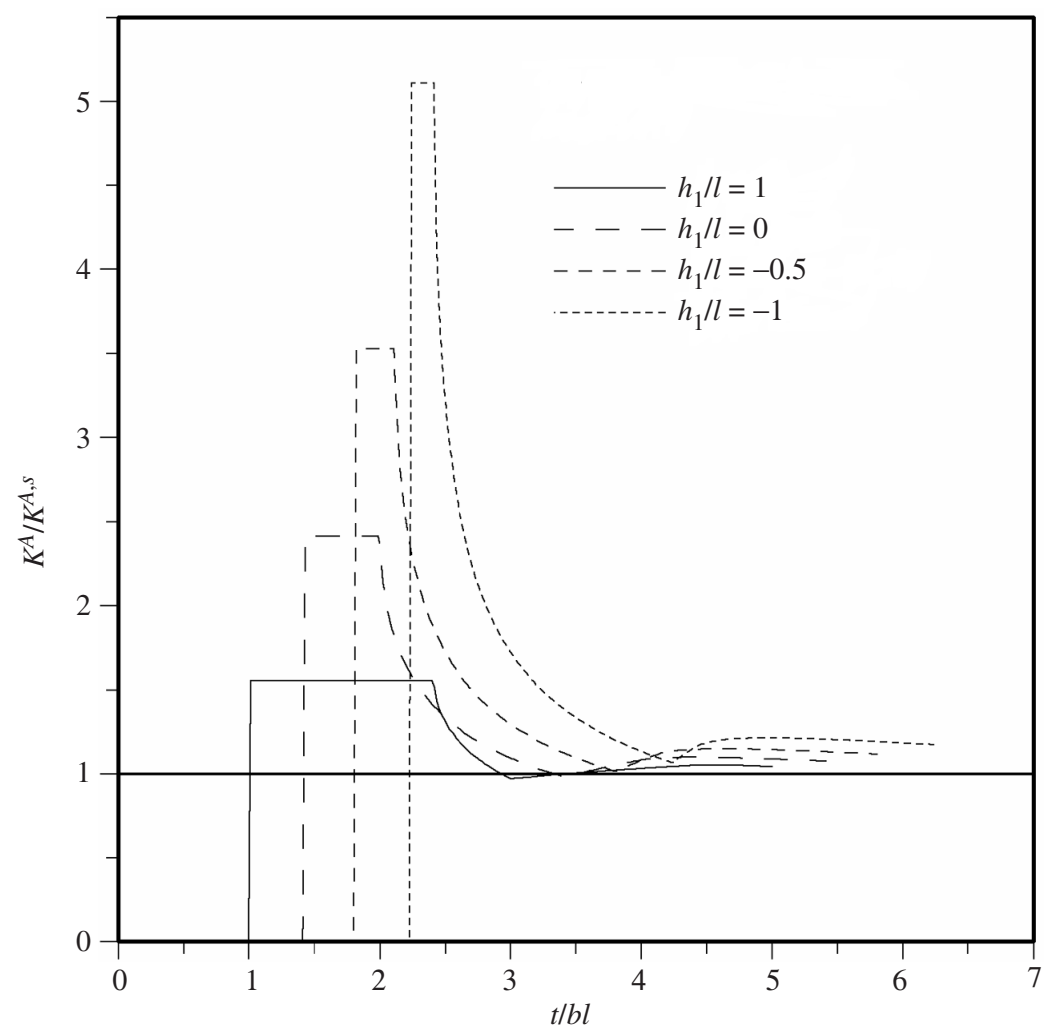

Figure 10. Transient responses of dimensionless stress-intensity factor $K^{\mathrm{A}} / K^{\mathrm{A}, s}$

for applying impact loading at $h_{2} / l=1$ for different values of $h_{1} / l$. $C_{44}: C_{45}: C_{55}=1: 1: 2$.

ation for detecting cracks. This study provides complete and exact information for the problem of a finite crack in an infinite medium subjected to dynamic loadings. Numerical calculations are performed to investigate the transient behaviour of shear stress and dynamic stress-intensity factor and the results are discussed in detail. The numerical calculations provided in this study can be seen as benchmark results for the transient analysis of finite cracks. The characteristic time in which the transient solution would approach the correspondent static value is analysed. It is found in this study that the transient effect can be neglected after the first few waves have passed. The ratio of the value for the maximum dynamic overshoot of the stress-intensity factor to the static value is expressed in a simple explicit form.

The authors gratefully acknowledge the financial support of this research by the National Science Council (Republic of China) under Grant NSC 88-2212-E-032-015.

\section{Appendix A.}

The functions and parameters of complete transient solutions for displacement and shear stresses presented in (3.33)-(3.35) are

$$
W^{\mathrm{i}}(X, Y, t)=\frac{P}{\pi C_{e}} \ln \left[\frac{t}{b R_{i}}+\left(\frac{t}{b R_{i}}\right)^{2}-1\right] \mathcal{H}\left(t-b R_{i}\right),
$$

Proc. R. Soc. A (2005) 


$$
\begin{aligned}
& \tau_{Y Z}^{\mathrm{i}}(X, Y, t)=\frac{-\operatorname{sgn}\left(Y-H_{2}\right) P t \sin \left(\Phi_{i}\right)}{\pi R_{i}\left(t^{2}-b^{2} R_{i}^{2}\right)^{1 / 2}} \mathcal{H}\left(t-b R_{i}\right), \\
& \tau_{X Z}^{\mathrm{i}}(X, Y, t)=\frac{-P t \cos \left(\Phi_{i}\right)}{\pi R_{i}\left(t^{2}-b^{2} R_{i}^{2}\right)^{1 / 2}} \mathcal{H}\left(t-b R_{i}\right), \\
& W^{r}(X, Y, t)=\frac{\operatorname{sgn}(Y) P}{\pi C_{e}} \ln \left[\frac{t}{b R_{r}}+\left(\frac{t}{b R_{r}}\right)^{2}-1\right] \\
& \times \mathcal{H}\left(t-b R_{r}\right) \mathcal{H}\left(\operatorname{sgn}(Y) \Phi-\pi+\Phi_{\mathrm{A}}\right) \mathcal{H}\left(\operatorname{sgn}\left(Y^{\prime}\right) \Phi^{\prime}-\pi+\Phi_{\mathrm{B}}\right), \\
& \tau_{Y Z}^{r}(X, Y, t)=-\frac{P t \sin \left(\Phi_{r}\right)}{\pi R_{r}\left(t^{2}-b^{2} R_{r}^{2}\right)^{1 / 2}} \mathcal{H}\left(t-b R_{r}\right) \\
& \times \mathcal{H}\left(\operatorname{sgn}(Y) \Phi-\pi+\Phi_{\mathrm{A}}\right) \mathcal{H}\left(\operatorname{sgn}\left(Y^{\prime}\right) \Phi^{\prime}-\pi+\Phi_{\mathrm{B}}\right), \\
& \tau_{X Z}^{r}(X, Y, t)=-\frac{\operatorname{sgn}(Y) P t \cos \left(\Phi_{r}\right)}{\pi R_{r}\left(t^{2}-b^{2} R_{r}^{2}\right)^{1 / 2}} \mathcal{H}\left(t-b R_{r}\right) \\
& \times \mathcal{H}\left(\operatorname{sgn}(Y) \Phi-\pi+\Phi_{\mathrm{A}}\right) \mathcal{H}\left(\operatorname{sgn}\left(Y^{\prime}\right) \Phi^{\prime}-\pi+\Phi_{\mathrm{B}}\right), \\
& W^{\mathrm{A}_{1}}(X, Y, t)=\frac{-\operatorname{sgn}(Y) P}{2 \pi^{2} C_{e}} \\
& \times \int_{b R+b R_{\mathrm{A}}}^{t} \int_{b R_{\mathrm{A}}}^{\tau-b R} \operatorname{Re}\left[\frac{\left(\partial \eta_{1}^{+} / \partial t_{1}\right)\left(\partial \eta_{2}^{+} / \partial t_{2}\right)}{\alpha_{+}\left(\eta_{1}^{+}\right)\left(\eta_{1}^{+}-\eta_{2}^{+}\right) \alpha_{-}\left(\eta_{2}^{+}\right)}\right. \\
& \left.-\frac{\left(\partial \eta_{1}^{-} / \partial t_{1}\right)\left(\partial \eta_{2}^{+} / \partial t_{2}\right)}{\alpha_{+}\left(\eta_{1}^{-}\right)\left(\eta_{1}^{-}-\eta_{2}^{+}\right) \alpha_{-}\left(\eta_{2}^{+}\right)}\right]_{t=\tau} \mathrm{d} t_{1} \mathrm{~d} \tau \\
& \tau_{Y Z}^{\mathrm{A}_{1}}(X, Y, t)=\frac{P}{2 \pi^{2}} \int_{b R_{\mathrm{A}}}^{t-b R} \operatorname{Re}\left[\frac{\alpha_{+}\left(\eta_{2}^{+}\right)\left(\partial \eta_{1}^{+} / \partial t_{1}\right)\left(\partial \eta_{2}^{+} / \partial t_{2}\right)}{\alpha_{+}\left(\eta_{1}^{+}\right)\left(\eta_{1}^{+}-\eta_{2}^{+}\right)}\right. \\
& \left.-\frac{\alpha_{+}\left(\eta_{2}^{+}\right)\left(\partial \eta_{1}^{-} / \partial t_{1}\right)\left(\partial \eta_{2}^{+} / \partial t_{2}\right)}{\alpha_{+}\left(\eta_{1}^{-}\right)\left(\eta_{1}^{-}-\eta_{2}^{+}\right)}\right] \mathrm{d} t_{1}, \\
& \tau_{X Z}^{\mathrm{A}_{1}}(X, Y, t)=\frac{-\operatorname{sgn}(Y) P}{2 \pi^{2}} \int_{b R_{\mathrm{A}}}^{t-b R} \operatorname{Re}\left[\frac{\eta_{2}^{+}\left(\partial \eta_{1}^{+} / \partial t_{1}\right)\left(\partial \eta_{2}^{+} / \partial t_{2}\right)}{\alpha_{+}\left(\eta_{1}^{+}\right)\left(\eta_{1}^{+}-\eta_{2}^{+}\right) \alpha_{-}\left(\eta_{2}^{+}\right)}\right. \\
& \left.-\frac{\eta_{2}^{+}\left(\partial \eta_{1}^{-} / \partial t_{1}\right)\left(\partial \eta_{2}^{+} / \partial t_{2}\right)}{\alpha_{+}\left(\eta_{1}^{-}\right)\left(\eta_{1}^{-}-\eta_{2}^{+}\right) \alpha_{-}\left(\eta_{2}^{+}\right)}\right] \mathrm{d} t_{1}, \\
& W^{\prime \mathrm{B}_{1}}\left(X^{\prime}, Y^{\prime}, t\right)=\frac{\operatorname{sgn}\left(Y^{\prime}\right) P}{2 \pi^{2} C_{e}} \\
& \times \int_{b R^{\prime}+b R_{\mathrm{B}}}^{t} \int_{b R_{\mathrm{B}}}^{\tau-b R^{\prime}} \operatorname{Re}\left[\frac{\left(\partial \eta_{1}^{+} / \partial t_{1}\right)\left(\partial \eta_{2}^{+} / \partial t_{2}\right)}{\alpha_{+}\left(\eta_{1}^{+}\right)\left(\eta_{1}^{+}-\eta_{2}^{+}\right) \alpha_{-}\left(\eta_{2}^{+}\right)}\right. \\
& \left.-\frac{\left(\partial \eta_{1}^{-} / \partial t_{1}\right)\left(\partial \eta_{2}^{+} / \partial t_{2}\right)}{\alpha_{+}\left(\eta_{1}^{-}\right)\left(\eta_{1}^{-}-\eta_{2}^{+}\right) \alpha_{-}\left(\eta_{2}^{+}\right)}\right]_{t=\tau} \mathrm{d} t_{1} \mathrm{~d} \tau
\end{aligned}
$$

Proc. R. Soc. A (2005) 
Finite crack anti-plane loadings

$$
\begin{gathered}
\tau_{Y^{\prime} Z^{\prime}}^{\mathrm{B}_{1}}\left(X^{\prime}, Y^{\prime}, t\right)=\frac{-P}{2 \pi^{2}} \int_{b R_{\mathrm{B}}}^{t-b R^{\prime}} \operatorname{Re}\left[\frac{\alpha_{+}\left(\eta_{2}^{+}\right)\left(\partial \eta_{1}^{+} / \partial t_{1}\right)\left(\partial \eta_{2}^{+} / \partial t_{2}\right)}{\alpha_{+}\left(\eta_{1}^{+}\right)\left(\eta_{1}^{+}-\eta_{2}^{+}\right)}\right. \\
\left.-\frac{\alpha_{+}\left(\eta_{2}^{+}\right)\left(\partial \eta_{1}^{-} / \partial t_{1}\right)\left(\partial \eta_{2}^{+} / \partial t_{2}\right)}{\alpha_{+}\left(\eta_{1}^{-}\right)\left(\eta_{1}^{-}-\eta_{2}^{+}\right)}\right] \mathrm{d} t_{1}, \\
\tau_{X^{\prime} Z^{\prime}}^{\mathrm{B}_{1}}\left(X^{\prime}, Y^{\prime}, t\right)=\frac{\operatorname{sgn}\left(Y^{\prime}\right) P}{2 \pi^{2}} \int_{b R_{\mathrm{B}}}^{t-b R^{\prime}} \operatorname{Re}\left[\frac{\eta_{2}^{+}\left(\partial \eta_{1}^{+} / \partial t_{1}\right)\left(\partial \eta_{2}^{+} / \partial t_{2}\right)}{\alpha_{+}\left(\eta_{1}^{+}\right)\left(\eta_{1}^{+}-\eta_{2}^{+}\right) \alpha_{-}\left(\eta_{2}^{+}\right)}\right. \\
\left.-\frac{\eta_{2}^{+}\left(\partial \eta_{1}^{-} / \partial t_{1}\right)\left(\partial \eta_{2}^{+} / \partial t_{2}\right)}{\alpha_{+}\left(\eta_{1}^{-}\right)\left(\eta_{1}^{-}-\eta_{2}^{+}\right) \alpha_{-}\left(\eta_{2}^{+}\right)}\right] \mathrm{d} t_{1}, \\
W^{\mathrm{A}_{n}}(X, Y, t)=\frac{\operatorname{sgn}(Y) P(i)^{q}}{2 \pi^{n+1}(i)^{n+1}} \int_{b R+b R_{0}+(n-1) b l}^{t} \int_{b R_{0}}^{\tau-b R-(n-1) b l} \int_{b l}^{a_{1}} \int_{b l}^{a_{2}} \\
\cdots \int_{b l}^{a_{n-1}} \mathrm{OP}[\mathrm{DISPL}]_{t=\tau} \mathrm{d} t_{n} \mathrm{~d} t_{n-1} \mathrm{~d} t_{n-2} \cdots \mathrm{d} t_{1} \mathrm{~d} \tau,
\end{gathered}
$$$$
\text { for } n=2,3,4, \ldots \text {, }
$$$$
\begin{aligned}
\tau_{Y Z}^{\mathrm{A}_{n}}(X, Y, t)=\frac{-P(i)^{q}}{2 \pi^{n+1}(i)^{n+1}} \int_{b R_{0}}^{t-b R-(n-1) b l} \int_{b l}^{b_{1}} \int_{b l}^{b_{2}} \\
\cdots \int_{b l}^{b_{n-1}} \mathrm{OP}[\mathrm{TAUYZ}] \mathrm{d} t_{n} \mathrm{~d} t_{n-1} \mathrm{~d} t_{n-2} \cdots \mathrm{d} t_{1},
\end{aligned}
$$$$
\text { for } n=2,3,4, \ldots \text {, }
$$

$$
\begin{gathered}
\tau_{X Z}^{\mathrm{A}_{n}}(X, Y, t)=\frac{\operatorname{sgn}(Y) P(i)^{q}}{2 \pi^{n+1}(i)^{n+1}} \int_{b R_{0}}^{t-b R-(n-1) b l} \int_{b l}^{b_{1}} \int_{b l}^{b_{2}} \\
\cdots \int_{b l}^{b_{n-1}} \operatorname{OP}[\text { TAUXZ }] \mathrm{d} t_{n} \mathrm{~d} t_{n-1} \mathrm{~d} t_{n-2} \cdots \mathrm{d} t_{1}
\end{gathered}
$$

for $n=2,3,4, \ldots$,

$$
\begin{array}{r}
W^{\prime \mathrm{B}_{n}}\left(X^{\prime}, Y^{\prime}, t\right)=\frac{-\operatorname{sgn}\left(Y^{\prime}\right) P(i)^{q}}{2 \pi^{n+1}(i)^{n+1}} \int_{b R^{\prime}+b R_{0}+(n-1) b l}^{t} \int_{b R_{0}}^{\tau-b R^{\prime}-(n-1) b l} \int_{b l}^{a_{1}^{\prime}} \int_{b l}^{a_{2}^{\prime}} \\
\cdots \int_{b l}^{a_{n-1}^{\prime}} \mathrm{OP}[\mathrm{DISPL}]_{t=\tau} \mathrm{d} t_{n} \mathrm{~d} t_{n-1} \mathrm{~d} t_{n-2} \cdots \mathrm{d} t_{1} \mathrm{~d} \tau
\end{array}
$$

for $n=2,3,4, \ldots$,

$$
\begin{gathered}
\tau_{Y^{\prime} Z^{\prime}}^{\mathrm{B}_{n}}\left(X^{\prime}, Y^{\prime}, t\right)=\frac{P(i)^{q}}{2 \pi^{n+1}(i)^{n+1}} \int_{b R_{0}}^{t-b R^{\prime}-(n-1) b l} \int_{b l}^{b_{1}^{\prime}} \int_{b l}^{b_{2}^{\prime}} \\
\cdots \int_{b l}^{b_{n-1}^{\prime}} \text { OP }[\text { TAUYZ }] \mathrm{d} t_{n} \mathrm{~d} t_{n-1} \mathrm{~d} t_{n-2} \cdots \mathrm{d} t_{1},
\end{gathered}
$$

for $n=2,3,4, \ldots$,

Proc. R. Soc. A (2005) 


$$
\begin{gathered}
\tau_{X^{\prime} Z^{\prime}}^{\mathrm{B}_{n}}\left(X^{\prime}, Y^{\prime}, t\right)=\frac{-\operatorname{sgn}\left(Y^{\prime}\right) P(i)^{q}}{2 \pi^{n+1}(i)^{n+1}} \int_{b R_{0}}^{t-b R^{\prime}-(n-1) b l} \int_{b l}^{b_{1}^{\prime}} \int_{b l}^{b_{2}^{\prime}} \\
\cdots \int_{b l}^{b_{n-1}^{\prime}} \text { OP }[\text { TAUXZ }] \mathrm{d} t_{n} \mathrm{~d} t_{n-1} \mathrm{~d} t_{n-2} \cdots \mathrm{d} t_{1},
\end{gathered}
$$

for $n=2,3,4, \ldots$,

in which

$$
\begin{aligned}
& a_{v}=\tau-b R-(n-v-1) b l-\left(t_{1}+t_{2}+\cdots+t_{v}\right), \quad \text { for } v=1,2, \ldots, n-1, \\
& b_{v}=t-b R-(n-v-1) b l-\left(t_{1}+t_{2}+\cdots+t_{v}\right), \quad \text { for } v=1,2, \ldots, n-1 \text {, } \\
& a_{v}^{\prime}=\tau-b R^{\prime}-(n-v-1) b l-\left(t_{1}+t_{2}+\cdots+t_{v}\right), \quad \text { for } v=1,2, \ldots, n-1 \text {, } \\
& b_{v}^{\prime}=t-b R^{\prime}-(n-v-1) b l-\left(t_{1}+t_{2}+\cdots+t_{v}\right), \quad \text { for } v=1,2, \ldots, n-1 \text {, } \\
& t_{1}+t_{2}+\cdots+t_{n+1}=t, \\
& G\left(\eta_{1}^{+}, \eta_{2}^{+}\right) G\left(\eta_{2}^{+}, \eta_{3}^{+}\right) G\left(\eta_{3}^{+}, \eta_{4}^{+}\right) \\
& \text {DISPL }=\frac{\cdots G\left(\eta_{n-1}^{+}, \eta_{n}^{+}\right)\left(\partial \eta_{1}^{+} / \partial t_{1}\right)\left(\partial \eta_{2}^{+} / \partial t_{2}\right)\left(\partial \eta_{3}^{+} / \partial t_{3}\right) \cdots\left(\partial \eta_{n+1}^{+} / \partial t_{n+1}\right)}{C_{e} \alpha_{+}\left(\eta_{1}^{+}\right)\left(\eta_{n}^{+}-\eta_{n+1}^{+}\right) \alpha_{-}\left(\eta_{n+1}^{+}\right)} \\
& G\left(\eta_{1}^{-}, \eta_{2}^{+}\right) G\left(\eta_{2}^{+}, \eta_{3}^{+}\right) G\left(\eta_{3}^{+}, \eta_{4}^{+}\right) \\
& -\frac{\cdots G\left(\eta_{n-1}^{+}, \eta_{n}^{+}\right)\left(\partial \eta_{1}^{-} / \partial t_{1}\right)\left(\partial \eta_{2}^{+} / \partial t_{2}\right)\left(\partial \eta_{3}^{+} / \partial t_{3}\right) \cdots\left(\partial \eta_{n+1}^{+} / \partial t_{n+1}\right)}{C_{e} \alpha_{+}\left(\eta_{1}^{-}\right)\left(\eta_{n}^{+}-\eta_{n+1}^{+}\right) \alpha_{-}\left(\eta_{n+1}^{+}\right)}, \\
& \mathrm{TAUYZ}=\mathrm{DISPL} \times C_{e} \alpha\left(\eta_{n+1}^{+}\right), \\
& \text {TAUXZ }=\text { DISPL } \times C_{e} \eta_{n+1}^{+}, \\
& \eta_{1}^{ \pm}= \begin{cases}\frac{t_{1} \cos \Phi_{\mathrm{A}}}{R_{\mathrm{A}}} \pm \mathrm{i} \frac{\sin \Phi_{\mathrm{A}}}{R_{\mathrm{A}}}\left(t_{1}^{2}-b^{2} R_{\mathrm{A}}^{2}\right)^{1 / 2}, & \text { for } A_{1}, A_{3}, A_{5}, \ldots ; B_{2}, B_{4}, B_{6} \ldots, \\
\frac{t_{1} \cos \Phi_{\mathrm{B}}}{R_{\mathrm{B}}} \pm \mathrm{i} \frac{\sin \Phi_{\mathrm{B}}}{R_{\mathrm{B}}}\left(t_{1}^{2}-b^{2} R_{\mathrm{B}}^{2}\right)^{1 / 2}, & \text { for } A_{2}, A_{4}, A_{6}, \ldots ; B_{1}, B_{3}, B_{5} \ldots,\end{cases} \\
& \eta_{v}^{ \pm}=\frac{-t_{v}}{l} \pm \mathrm{i} \varepsilon, \quad \text { for } v=2,3, \ldots, n, \quad \text { for } A_{n} \text { and } B_{n} \text { waves }(n \geqslant 2) \text {, } \\
& \eta_{n+1}^{ \pm}= \begin{cases}\frac{-t_{n+1} \cos \Phi}{R} \pm \mathrm{i} \frac{\sin \Phi}{R}\left(t_{n+1}^{2}-b^{2} R^{2}\right)^{1 / 2}, & \text { for } A_{n} \text { waves } \\
\frac{-t_{n+1} \cos \Phi^{\prime}}{R^{\prime}} \pm \mathrm{i} \frac{\sin \Phi^{\prime}}{R^{\prime}}\left(t_{n+1}^{2}-b^{2} R^{\prime 2}\right)^{1 / 2}, & \text { for } B_{n} \text { waves }\end{cases} \\
& \mathrm{OP}= \begin{cases}\operatorname{Im}, \quad q=1, R_{0}=R_{\mathrm{B}}, & \text { when } n=2,4,6, \ldots, \\
\operatorname{Re}, \quad q=0, R_{0}=R_{\mathrm{A}}, & \text { when } n=3,5,7, \ldots,\end{cases} \\
& R_{i}=\left[\left(X-H_{1}\right)^{2}+\left(Y-H_{2}\right)^{2}\right]^{1 / 2}, \quad \Phi_{i}=\arccos \left(\frac{X-H_{1}}{R_{i}}\right),
\end{aligned}
$$

Proc. R. Soc. A (2005) 


$$
\begin{array}{lc}
R_{r}=\left[\left(X-H_{1}\right)^{2}+\left(|Y|+H_{2}\right)^{2}\right]^{1 / 2}, & \Phi_{r}=\arccos \left(\frac{X-H_{1}}{R_{r}}\right), \\
R_{\mathrm{A}}=\left(H_{1}^{2}+H_{2}^{2}\right)^{1 / 2}, & \Phi_{\mathrm{A}}=\arccos \left(\frac{H_{1}}{R_{\mathrm{A}}}\right), \\
R_{\mathrm{B}}=\left(H_{1}^{\prime 2}+H_{2}^{\prime 2}\right)^{1 / 2}, & \Phi_{\mathrm{B}}=\arccos \left(\frac{H_{1}^{\prime}}{R_{\mathrm{B}}}\right), \\
R=\left(X^{2}+Y^{2}\right)^{1 / 2}, & \Phi=\operatorname{sgn}(Y) \arccos \left(\frac{X}{R}\right), \\
R^{\prime}=\left(X^{\prime 2}+Y^{\prime 2}\right)^{1 / 2}, & \Phi^{\prime}=\operatorname{sgn}\left(Y^{\prime}\right) \arccos \left(\frac{X^{\prime}}{R^{\prime}}\right), \\
G\left(\eta_{1}, \eta_{2}\right)=\frac{\alpha_{-}\left(\eta_{2}\right)}{\left(\eta_{1}+\eta_{2}\right) \alpha_{-}\left(-\eta_{2}\right)} .
\end{array}
$$

\section{Appendix B.}

The functions and parameters of dynamic stress-intensity factor presented in (4.3) are

$$
\begin{aligned}
& c_{1}=\tau-(n-1) b l, \\
& c_{v}=\tau-(n-v) b l-\left(t_{1}+t_{2}+\cdots+t_{v-1}\right), \quad \text { for } v=2,3, \ldots, n-1, \\
& t_{1}+t_{2}+\cdots+t_{n}=t \\
& \mathrm{SIF}=G\left(\eta_{1}^{+}, \eta_{2}^{+}\right) G\left(\eta_{2}^{+}, \eta_{3}^{+}\right) \cdots G\left(\eta_{n-1}^{+}, \eta_{n}^{+}\right) \frac{\partial \eta_{1}^{+}}{\partial t_{1}} \frac{\partial \eta_{2}^{+}}{\partial t_{2}} \cdots \frac{\partial \eta_{n}^{+}}{\partial t_{n}}\left\{\alpha_{+}\left(\eta_{1}^{+}\right)\right\}^{-1} \\
& -G\left(\eta_{1}^{-}, \eta_{2}^{+}\right) G\left(\eta_{2}^{+}, \eta_{3}^{+}\right) \cdots G\left(\eta_{n-1}^{+}, \eta_{n}^{+}\right) \frac{\partial \eta_{1}^{-}}{\partial t_{1}} \frac{\partial \eta_{2}^{+}}{\partial t_{2}} \cdots \frac{\partial \eta_{n}^{+}}{\partial t_{n}}\left\{\alpha_{+}\left(\eta_{1}^{-}\right)\right\}^{-1}, \\
& \eta_{1}^{ \pm}=\frac{t_{1} \cos \Phi_{0}}{R_{0}} \pm \mathrm{i} \frac{\sin \Phi_{0}}{R_{0}}\left(t_{1}^{2}-b^{2} R_{0}^{2}\right)^{1 / 2}, \quad \eta_{v}^{ \pm}=\frac{-t_{v}}{l} \pm \mathrm{i} \varepsilon, \quad \text { for } v=2,3, \ldots, n, \\
& \mathrm{OP}= \begin{cases}\operatorname{Re}, \quad q=0, R_{0}=R_{\mathrm{B}}, \Phi_{0}=\Phi_{\mathrm{B}}, & \text { when } n=2,4,6, \ldots, \\
\operatorname{Im}, \quad q=1, R_{0}=R_{\mathrm{A}}, \Phi_{0}=\Phi_{\mathrm{A}}, & \text { when } n=3,5,7, \ldots,\end{cases} \\
& R_{\mathrm{A}}=\left(H_{1}^{2}+H_{2}^{2}\right)^{1 / 2}, \quad \Phi_{\mathrm{A}}=\arccos \left(\frac{H_{1}}{R_{\mathrm{A}}}\right), \\
& R_{\mathrm{B}}=\left(H_{1}^{\prime 2}+H_{2}^{\prime 2}\right)^{1 / 2}, \quad \Phi_{\mathrm{B}}=\arccos \left(\frac{H_{1}^{\prime}}{R_{\mathrm{B}}}\right) .
\end{aligned}
$$

\section{References}

Achenbach, J. D. 1973 Wave propagation in elastic solids. Amsterdam: North-Holland.

Brock, L. M. 1982 Shear and normal impact loading on one face of a narrow slit. Int. J. Solids Struct. 18, 467-477.

Proc. R. Soc. A (2005) 
Brock, L. M. 1984 Stresses in a surface obstacle undercut due to rapid indentation. J. Elasticity 14, 415-424.

Brock, L. M., Jolles, M. \& Schroedl, M. 1985 Dynamic impact over a subsurface crack: applications to the dynamic tear test. J. Appl. Mech. 52, 287-290.

Cherkaev, A., Lurie, K. \& Milton, G. W. 1992 Invariant properties of stress in plane elasticity and equivalent classes of composites. Proc. R. Soc. A 438, 519-529.

Das, S. \& Patra, B. 1996 Moving Griffith crack at the interface of two dissimilar orthotropic half planes. Engng Fract. Mech. 54, 523-531.

Das, S. \& Patra, B. 1998 Stress intensity factors for moving interfacial crack between bonded dissimilar fixed orthotropic layers. Computers Struct. 69, 459-472.

Dundurs, J. \& Markenscoff, X. 1993 Invariance of stresses under a change in elastic compliances. Proc. R. Soc. A 443, 289-300.

de Hoop, A. T. 1958 Representation theorems for the displacement in an elastic solid and their application to elastodynamic diffraction theory. Doctoral dissertation, Technische Hogeschool, Delft.

Flitman, L. M. 1963 Waves generated by sudden crack in a continuous elastic medium. Appl. Math. Mech. 27, 938-953.

Freund, L. B. 1974 The stress intensity factor due to normal impact loading of the faces of a crack. Int. J. Engng Sci. 12, 179-189.

Freund, L. B. 1990 Dynamic fracture mechanics. Cambridge University Press.

Ing, Y. S. \& Ma, C. C. 1996 Transient response of a finite crack subjected to dynamic anti-plane loading. Int. J. Fracture 82, 345-362.

Ing, Y. S. \& Ma, C. C. 1997 Dynamic fracture analysis of a finite crack subjected to an incident horizontally polarized shear wave. Int. J. Solids Struct. 34, 895-910.

Itou, S. \& Haliding, H. 1997 Dynamic stress intensity factors around two parallel cracks in an infinite-orthotropic plane subjected to incident harmonic stress waves. Int. J. Solids Struct. 34, 1145-1165.

Kassir, M. K. \& Bandyopadhyay, K. K. 1983 Impact response of a cracked orthotropic medium. J. Appl. Mech. 50, 630-636.

Kostrov, B. V. 1964 Self-similar problems of propagation of shear cracks. Appl. Math. Mech. 28, 1077-1087.

Lin, R. L. \& Ma, C. C. 2000 Antiplane deformations for anisotropic multilayered media by using the coordinate transform method. J. Appl. Mech. 67, 597-605.

Liu, G. R. \& Achenbach, J. D. 1994 A strip element method for stress analysis of anisotropic linearly elastic solids. J. Appl. Mech. 61, 270-277.

Liu, G. R. \& Achenbach, J. D. 1995 Strip element method to analyze wave scattering by cracks in anisotropic laminated plates. J. Appl. Mech. 62, 607-613.

Liu, G. R., Xi, Z. C., Lam, K. Y. \& Shang, H. M. 1999 A strip element method for analyzing wave scattering by a crack in an immersed composite laminate. J. Appl. Mech. 66, 898-903.

Loeber, J. F. \& Sih, G. C. 1968 Diffraction of antiplane shear waves by a finite crack. J. Acoust. Soc. Am. 44, 90-98.

Ma, C. C. 1996 Relationship of anisotropic and isotropic materials for antiplane problems. AIAA J. 34, 2453-2456.

Ma, C. C. \& Chen, S. K. 1994 Exact transient full-field analysis of an anti-plane subsurface crack subjected to dynamic impact loading. J. Appl. Mech. 61, 649-655.

Ma, C. C. \& Hou, Y. C. 1990 Theoretical analysis of the transient response for a stationary in-plane crack subjected to dynamic impact loading. Int. J. Engng Sci. 28, 1321-1329.

Ma, C. C. \& Hou, Y. C. 1991 Transient analysis for anti-plane crack subjected to dynamic loadings. J. Appl. Mech. 58, 703-709.

Ma, C. C. \& Liao, C. Y. 1999 Elastodynamic analysis of antiplane anisotropic interface cracks. J. Engng Mech. 125, 891-898.

Proc. R. Soc. A (2005) 
Noble, B. 1958 Methods based on the Wiener-Hopf technique. Elmsford, NY: Pergamon.

Pramanik, R. K., Pal, S. C. \& Ghosh, M. L. 1999 High frequency scattering due to a pair of time-harmonic antiplane forces on the faces of a finite interface crack between dissimilar anisotropic materials. Eur. J. Mech. A 18, 1013-1026.

Rizza, R. \& Nair, S. 1999 A penny shaped crack in a transversely isotropic material under non-axisymmetric impact loads. Int. J. Solids Struct. 36, 35-64.

Rubio-Gonzalez, C. \& Mason, J. J. 1999 Response of finite cracks in orthotropic materials due to concentrated impact shear loads. J. Appl. Mech. 66, 485-491.

Rubio-Gonzalez, C. \& Mason, J. J. 2000 Dynamic stress intensity factors at the tip of a uniformly loaded semi-infinite crack in an orthotropic material. J. Mech. Phys. Solids 48, 899-925.

Rubio-Gonzalez, C. \& Mason, J. J. 2001 Green's functions for the stress intensity factor evolution in finite cracks in orthotropic materials. Int. J. Fracture 108, 317-335.

Sarkar, J., Mandal, S. C. \& Ghosh, M. L. 1995 Diffraction of elastic waves by three coplanar griffith cracks in an orthotropic medium. Int. J. Engng Sci. 33, 163-177.

Shen, S. \& Kuang, Z. B. 1998 Wave scattering from an interface crack in laminated anisotropic media. Mech. Res. Commun. 25, 509-517.

Shindo, Y. \& Nozaki, H. 1991 Impact response of a finite crack in an orthotropic strip. Acta Mech. 62, 87-104.

Sih, G. C. \& Embley, G. T. 1972 Impact response of a finite crack in plane extension. Int. J. Solids Struct. 8, 977-993.

Sih, G. C. \& Loeber, J. F. 1969 Wave propagation in an elastic solid with a line of discontinuity or finite crack. Q. Appl. Math. 27, 193-213.

Thau, S. A. \& Lu, T. H. 1971 Transient stress intensity factor for a finite crack in an elastic solid caused by a dilatational wave. Int. J. Solids Struct. 7, 731-750.

Ting, T. C. T. 1995 Generalized Dundurs constants for anisotropic bimaterials. Int. J. Solids Struct. 32, 483-500.

Ting, T. C. T. 1996 Anisotropic elasticity: theory and applications. Oxford University Press.

Ting, T. C. T. 2000 Recent developments in anisotropic elasticity. Int. J. Solids Struct. 37, 401-409.

Tsai, C. H. \& Ma, C. C. 1992 Transient analysis of a semi-infinite crack subjected to dynamic concentrated forces. J. Appl. Mech. 59, 804-811.

Tsai, C. H. \& Ma, C. C. $1997 a$ Transient analysis of a propagating in-plane crack in a finite geometry body subjected to static loadings. J. Appl. Mech. 64, 620-628.

Tsai, C. H. \& Ma, C. C. $1997 b$ Theoretical transient analysis of the interaction between a dynamically propagating in-plane crack and traction free boundaries. J. Appl. Mech. 64, 819-827.

Xi, Z. C., Liu, G. R., Lam, K. Y. \& Shang, H. M. 2000 A strip-element method for analyzing wave scatterning by a crack in a fluid-filled composite cylindrical shell. Compos. Sci. Tech. 60, 1985-1996.

Yang, W. \& Ma, C. C. 1998 Orthotropic transform for planar anisotropic elasticity and reduced dependence of elastic constants. Proc. R. Soc. A 454, 1843-1855.

Zhang, C. 2000 Transient elastodynamic antiplane crack analysis of anisotropic solids. Int. J. Solids Struct. 37, 6107-6130.

Zhao, X. 2001 The stress-intensity factor history for a half plane crack in a transversely isotropic solid due to impact point loading on the crack faces. Int. J. Solids Struct. 38, 2851-2865. 